\title{
Geochemistry of the dissolved loads during high-flow season of rivers in the southeastern coastal region of China: anthropogenic impact on chemical weathering and carbon sequestration
}

\author{
Wenjing Liu ${ }^{1,2,3}$, Zhifang $\mathrm{Xu}^{1,2,3}$, Huiguo Sun ${ }^{1,3}$, Tong $\mathrm{Zhao}^{1,3}$, Chao Shi ${ }^{3}$, and Taoze $\mathrm{Liu}^{4}$ \\ ${ }^{1}$ Key Laboratory of Cenozoic Geology and Environment, Institute of Geology and Geophysics, Institutions of Earth Science, \\ Chinese Academy of Sciences, Beijing 100029, China \\ ${ }^{2}$ CAS Center for Excellence in Life and Paleoenvironment, Beijing, 100044, China \\ ${ }^{3}$ University of Chinese Academy of Sciences, Beijing 100049, China \\ ${ }^{4}$ State Key Laboratory of Environmental Geochemistry, Institute of Geochemistry, Chinese Academy of Sciences, \\ Guiyang, Guizhou 550002, China
}

Correspondence: Zhifang Xu (zfxu@mail.iggcas.ac.cn)

Received: 27 February 2018 - Discussion started: 20 March 2018

Revised: 4 July 2018 - Accepted: 27 July 2018 - Published: 22 August 2018

\begin{abstract}
The southeastern coastal region is one of the most developed and populated areas in China. Meanwhile, it has been severely impacted by acid rain over many years. The chemical compositions and carbon isotope compositions of dissolved inorganic carbon $\left(\delta^{13} \mathrm{C}_{\mathrm{DIC}}\right)$ in river water in the high-flow season were investigated to estimate the chemical weathering and associated atmospheric $\mathrm{CO}_{2}$ consumption rates as well as the acid-deposition disturbance. Mass balance calculations indicated that the dissolved loads of major rivers in the Southeast Coastal River Basin (SECRB) were contributed to by atmospheric (14.3\%, $6.6 \%-23.4 \%)$, anthropogenic $(15.7 \%, 0 \%-41.1 \%)$, silicate weathering $(39.5 \%, 17.8 \%-74.0 \%)$ and carbonate weathering inputs $(30.6 \%, 3.9 \%-62.0 \%)$. The silicate and carbonate chemical weathering rates for these river watersheds were $14.2-35.8$ and $1.8-52.1 \mathrm{t} \mathrm{km}^{-2} \mathrm{a}^{-1}$, respectively. The associated mean $\mathrm{CO}_{2}$ consumption rate by silicate weathering for the whole SECRB was $191 \times 10^{3} \mathrm{~mol} \mathrm{~km}^{-2} \mathrm{a}^{-1}$. The chemical and $\delta^{13} \mathrm{C}_{\mathrm{DIC}}$ evidence indicated that sulfuric and nitric acid (mainly from acid deposition) were significantly involved in the chemical weathering of rocks. There was an overestimation of $\mathrm{CO}_{2}$ consumption at $0.19 \times 10^{12} \mathrm{~g} \mathrm{Ca}^{-1}$ if sulfuric and nitric acid were ignored, which accounted for about $33.6 \%$ of the total $\mathrm{CO}_{2}$ consumption by silicate weathering in the SECRB. This study quantitatively highlights the role of acid deposition in chemical weathering, suggesting that the anthropogenic impact should be seriously consid-
\end{abstract}

ered in estimations of chemical weathering and associated $\mathrm{CO}_{2}$ consumption.

\section{Introduction}

Chemical weathering of rocks is the key process that links geochemical cycling of solid earth to the atmosphere and ocean. It provides nutrients to terrestrial and marine ecosystems and regulates the level of atmospheric $\mathrm{CO}_{2}$. As a net sink of atmospheric $\mathrm{CO}_{2}$ on geologic timescales, estimations of silicate chemical weathering rates and the controlling factors are important issues that are related to long-term global climate change (e.g., Raymo and Ruddiman, 1992; Négrel et al. 1993; Berner and Caldeira, 1997; Gaillardet et al., 1999; Kump et al., 2000; Amiotte-Suchet et al., 2003; Oliva et al., 2003; Hartmann et al., 2009; Moon et al., 2014). As an important component in the Earth's critical zone (U.S. National Research Council Committee, 2001), rivers serve as integrators of various natural and anthropogenic processes and products in a basin, and as carriers, transporting the weathering products from the continent to the ocean. Therefore, the chemical compositions of river water are widely used to evaluate chemical weathering and associated $\mathrm{CO}_{2}$ consumption rates at catchment and/or continental scale and to examine their controlling factors (e.g., Edmond et al., 1995; Gisla- 
son et al., 1996; Galy and France-Lanord, 1999; Huh, 2003; Millot et al., 2002, 2003; Oliva et al., 2003; West et al., 2005; Moon et al., 2007; Noh et al., 2009; Shin et al., 2011; Calmels et al., 2011; Li et al., 2014).

With the intensification of human activities, human perturbations to river basins have increased in frequency and magnitude (Raymond et al., 2008; Regnier et al., 2013; Li and Bush, 2015). It is important to understand how such perturbations function in the current weathering systems and to predict how they will affect the critical zone of the future (Brantley and Lebedeva, 2011). In addition to $\mathrm{CO}_{2}$, other sources of acidity (such as sulfuric, nitric and organic acids) can produce protons. These protons react with carbonate and silicate minerals, thus enhancing rock chemical weathering rates and flux compared with only considering protons deriving from $\mathrm{CO}_{2}$ dissolution (Calmels et al., 2007; $\mathrm{Xu}$ and Liu, 2010). The effect of other sourced protons (especially $\mathrm{H}^{+}$ induced by $\mathrm{SO}_{2}$ and $\mathrm{NO}_{x}$ coming from anthropogenic activities) on chemical weathering is documented to be an important mechanism modifying atmospheric $\mathrm{CO}_{2}$ consumption by rock weathering (Galy and France-Lanord, 1999; Semhi et al., 2000; Spence and Telmer, 2005; Xu and Liu, 2007; Perrin et al., 2008; Gandois et al., 2011). Anthropogenic emissions of $\mathrm{SO}_{2}$ were projected to provide 3 to 5 times more $\mathrm{H}_{2} \mathrm{SO}_{4}$ to the continental surface than the pyrite oxidation (Lerman et al., 2007). Therefore, increasing acid precipitation due to intense human activity nowadays could make this mechanism more prominent.

The role that acid precipitation plays on chemical weathering and $\mathrm{CO}_{2}$ consumption has been investigated in some river catchments (Amiotte-Suchet et al., 1995; Probst et al., 2000; Vries et al., 2003; Lerman et al., 2007; Xu and Liu, 2010). It has been documented that silicate rocks were more easily disturbed by acid precipitation during their weathering and soil-leaching processes because of their low buffeting capacity (Reuss et al., 1987; Amiotte-Suchet et al., 1995). The disturbance could be intensive and cause a decrease in $\mathrm{CO}_{2}$ consumption by weathering by about $73 \%$ due to acid precipitation in the Strengbach catchment (Vosges Mountains, France), which is dominated by crystalline rocks (AmiotteSuchet et al., 1995). This highlights the importance of exploring the anthropogenic impact on chemical weathering and $\mathrm{CO}_{2}$ consumption under different backgrounds (e.g., lithology, climate, human activity intensity and basin scale) for better constraining and estimating the effect of acid precipitation on rock weathering. Asia, especially eastern Asia, is one of the world's major sulfur and nitrogen emission areas. However, the effect of acid precipitation on silicate weathering and associated $\mathrm{CO}_{2}$ consumption has not been well evaluated in this area, especially lacks quantitative studies.

Acid precipitation affected about $30 \%$ of the territory of China (Fig. 1), and the seriously polluted areas are mainly located in the east, south and center of China, where over $70 \%$ of the cities were suffering from acid rain (M. Zhang et al., 2007; Ministry of Environmental Protection of China,
2009). The southeastern coastal region of China is one of the most developed and populated areas of this country, dominated by Mesozoic magmatic rocks (mainly granite and volcanic rocks) in lithology. Meanwhile, the southeast coastal area has become one of the three major acid-rain areas in China since the beginning of 1990s (Larssen and Carmichael, 2000). It is seriously impacted by acid rain, with a volumeweighted mean value of $\mathrm{pH}$ lower than 4.5 for many years (Wang et al., 2000; Larssen and Carmichael, 2000; Zhao, 2004; Han et al., 2006; Larssen et al., 2006; M. Zhang et al., 2007; Huang et al., 2008; Xu et al., 2011). Therefore, it is an ideal area for evaluating silicate weathering and the associated acid-rain effects. In previous work, we have recognized and discussed the importance of sulfuric acid on rock weathering and associated $\mathrm{CO}_{2}$ consumption in the Qiantang river basin in this area (W. Liu et al., 2016). However, it is difficult to infer the anthropogenic impact on chemical weathering and $\mathrm{CO}_{2}$ consumption in the whole southeastern coastal area from the case study of a single river basin, because of the variations on lithology, basin scale, runoff and anthropogenic condition in the large acid-deposition-affected area. In this study, the chemical and carbon isotope composition of river water in this area were first systematically investigated in order to (i) decipher the different sources of solutes and to quantify their contributions to the dissolved loads, (ii) calculate silicate weathering and associated $\mathrm{CO}_{2}$ consumption rates, and (iii) evaluate the effects of acid deposition on rock weathering and $\mathrm{CO}_{2}$ consumption flux in the whole of the SECRB.

\section{Natural setting of study area}

The southeastern coastal region of China, where the landscape is dominated by mountainous and hilly terrain, lacks the conditions for developing large rivers. The rivers in this region have dominantly small- and medium-sized drainage areas due to the topographic limitation. Only five rivers in this region have lengths over $200 \mathrm{~km}$ and drainage areas over $10000 \mathrm{~km}^{2}$, and they are the Qiantangjiang (Qiantang) and the Oujiang $(\mathrm{Ou})$ in Zhejiang province, the Minjiang (Min) and the Jiulongjiang (Jiulong) in Fujian province and the Hanjiang (Han) in Guangdong province, from north to south (Fig. 1). Rivers in this region generally flow eastward or southward and finally inject into the East China Sea or the South China Sea (Fig. 1), and they are collectively named Southeast Coastal Rivers (SECRs).

The Southeast Coastal River Basin (SECRB) is in the warm and humid subtropical oceanic monsoon climate. The mean annual temperature and precipitation are $17-21^{\circ} \mathrm{C}$ and 1400-2000 mm, respectively. The precipitation mainly happens during May to September, and the lowest and highest temperatures often occur in January and July. This area is one of the most developed areas in China, with a population more than 190 million (mean density of $\sim 470$ individuals $\mathrm{km}^{-2}$ ), 


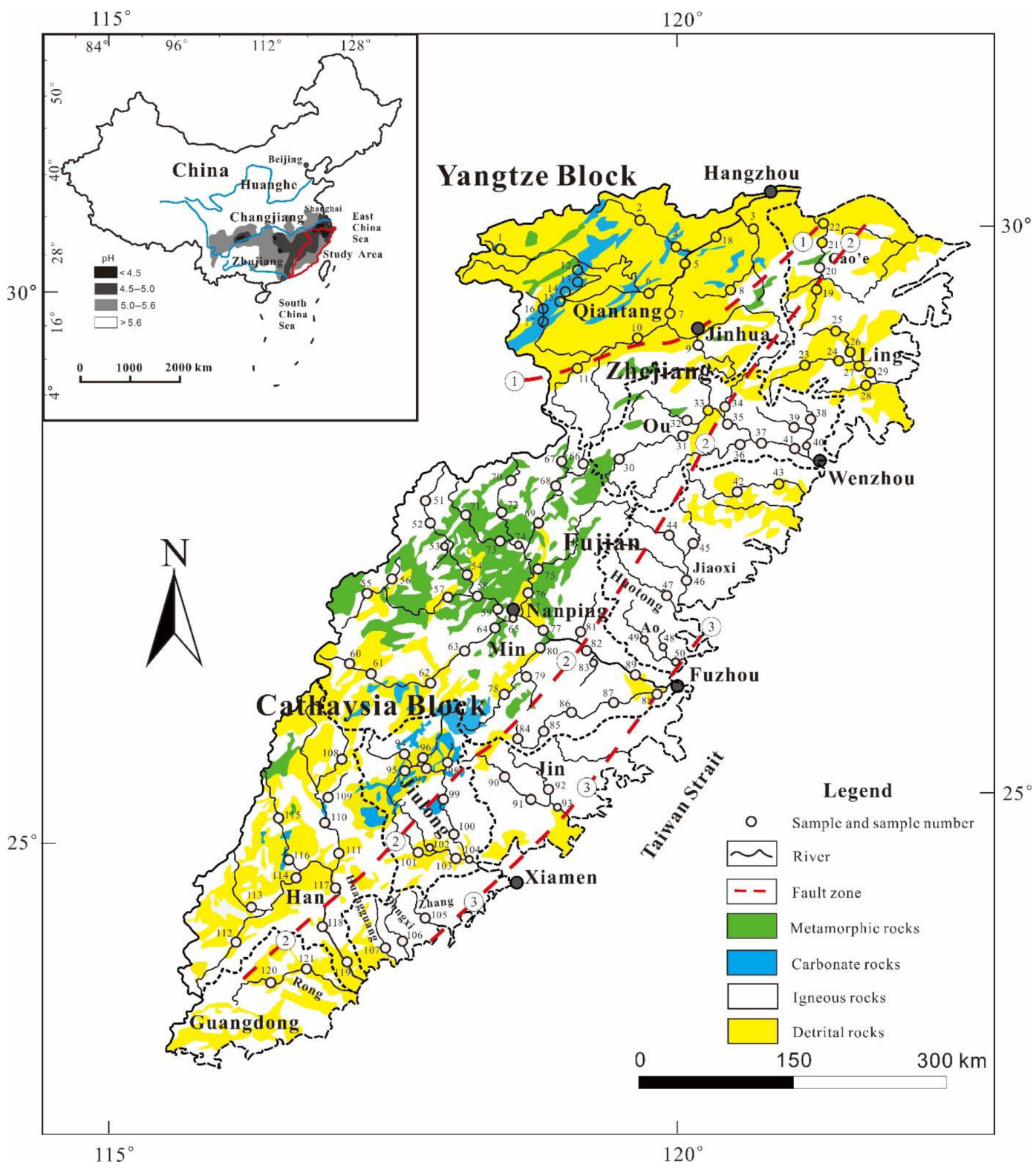

Figure 1. Sketch map showing the lithology, sampling locations and sample number of the SECR drainage basin, and regional rainwater pH ranges are shown in the sketch map in the upper-left (modified from Zhou and Li, 2000; Shu et al., 2009; Xu et al., 2016, rainwater acidity distribution of China mainland is from State Environmental Protection Administration of China). (1) Shaoxing-Jiangshan fault zone, (2) Zhenghe-Dapu fault zone and (3) Changle-Nanao fault zone. The figure was created by CorelDraw software version 17.1 .

but the population is mainly concentrated in the coastal urban areas. The vegetation coverage of these river basins is higher than $60 \%$, mainly subtropical evergreen-deciduous broadleaf forest and mostly distributed in mountainous areas. Cultivated land, industries and cities are mainly located in the plain areas and lower reaches of these rivers.

Geologically, three regional-scale fault zones are distributed across the SECRB region (Fig. 1). They are the sub-EW-trending Shaoxing-Jiangshan fault zone, the NEtrending Zhenghe-Dapu fault zone and the NE-trending Changle-Nanao fault zone (Shu et al., 2009). These fault zones dominate the direction of the mountains ridgelines and drainages, as well as the formation of the basins and bay. The Zhenghe-Dapu fault zone is a boundary line of the Caledonian uplift belt and Hercynian-Indonesian depression zone. Mesozoic magmatic rocks are widespread in the southeastern coastal region with a total outcrop area at about $240000 \mathrm{~km}^{2}$. Over $90 \%$ of the Mesozoic magmatic rocks are granitoids (granites and rhyolites), with a minor amount of basalt (Zhou and Li, 2000; Zhou et al., 2006; Bai et al., 2014). These crustderived granitic rocks are mainly formed in the Yanshanian stage and may have been related to multiple collision events 
between Cathaysia and Yangtze blocks and the Pacific plate (Zhou and Li, 2000; Xu et al., 2016). Among the major river basins, the proportions of magmatic rocks outcrop are about $36 \%$ in the Qiantang catchment, over $80 \%$ in the Ou, the Jiaoxi and the Jin catchments and around $60 \%$ in the Min, the Jiulong, the Han and the Rong catchments (Shi, 2014). The overlying Quaternary sediment in this area is composed of brown-yellow siltstones but is rarely developed. The oldest basement complex is composed of metamorphic rocks of greenschist and amphibolite facies. Sedimentary rocks are categorised into two types: one is mainly composed of red clastic rocks which cover more than $40000 \mathrm{~km}^{2}$ in the area and the other occurs as interlayers within volcanic formations, including varicolored mudstones and sandstones. They are mainly distributed on the west of Zhenghe-Dapu fault zone (FJBGMR, 1985; ZJBGMR, 1989; Shu et al., 2009).

\section{Sampling and analytical method}

A total of 121 water samples were collected from the major rivers and their tributaries in the SECRB in July of 2010 in the high-flow period (sample number and locations are shown in Fig. 1). For the river low-reach samples, the sampling sites were selected as far as possible from the tideimpacted area and the sampling was conducted during lowtide period (based on the daily tidal time, http://ocean.cnss. com.cn/, last access: 31 July 2010) on the sampling day. Besides, the salinity of the waters was checked by a salinometer (WS202, China) before sampling in the field. In addition, water chemistry data were double checked to make sure that the river samples were not contaminated by seawater. Water samples were collected in the middle channel of the rivers from bridges or ferries or directly from the center of some shallow streams. Temperature (T), $\mathrm{pH}$ and electrical conductivity (EC) were measured in the field with a portable $\mathrm{EC} / \mathrm{pH}$ meter (YSI-6920, USA). All of the water samples for chemical analysis were filtered in field through a $0.22 \mu \mathrm{m}$ Millipore membrane filter, and the first portion of the filtration was discarded to wash the membrane and filter. One portion of filtrate was stored directly in HDPE bottles for anion analysis and another was acidified to $\mathrm{pH}<2$ with $6 \mathrm{M}$ double sub-boiling distilled $\mathrm{HNO}_{3}$ for cation analysis. All containers were previously washed with high-purity $\mathrm{HCl}$ and rinsed with Milli-Q $18.2 \mathrm{M} \Omega$ water.

Alkalinity was determined by phenolphthalein and methyl orange end-point titration with dilute $\mathrm{HCl}$ within $12 \mathrm{~h}$ after sampling. The $\mathrm{HCl}$ consumption volumes for phenolphthalein and methyl orange end-point titration were used to calculate the $\mathrm{HCO}_{3}^{-}$. Cations $\left(\mathrm{Na}^{+}, \mathrm{K}^{+}, \mathrm{Ca}^{2+}\right.$ and $\mathrm{Mg}^{2+}$ ) were determined using an inductively coupled plasma atomic emission spectrometer (ICP-AES) (IRIS Intrepid II XSP, USA). Anions $\left(\mathrm{Cl}^{-}, \mathrm{F}^{-}, \mathrm{NO}_{3}^{-}\right.$and $\left.\mathrm{SO}_{4}^{2-}\right)$ were analyzed by ionic chromatography (IC) (Dionex Corporation, USA). Dissolved silica was determined by spectrophotometry with the molybdate blue method. Reagent and procedural blanks were measured parallel to the sample treatment, and the calibration curve was evaluated by quality control standards before, during and after the analyses of each batch of samples. Measurement reproducibility was determined by duplicated sample and standards, which showed $\pm 3 \%$ precision for the cations and $\pm 5 \%$ for the anions. Analyzing water chemistry was conducted in the hydrochemistry and environmental laboratory at the Institute of Geology and Geophysics, Chinese Academy of Sciences.

Samples for a carbon isotopic ratio $\left(\delta^{13} \mathrm{C}\right)$ of dissolved inorganic carbon (DIC) measurements were collected in $150 \mathrm{~mL}$ glass bottles with air-tight caps and preserved with $\mathrm{HgCl}_{2}$ to prevent biological activity. The samples were refrigerated until analysis. For the $\delta^{13} \mathrm{C}$ measurements, the filtered samples were injected into glass bottles with phosphoric acid. The $\mathrm{CO}_{2}$ was then extracted and cryogenically purified using a high vacuum line. $\delta^{13} \mathrm{C}$ isotopic ratios were analyzed on Finnigen MAT-252 stable isotope mass spectrometer at the State Key Laboratory of Environmental Geochemistry, Chinese Academy of Sciences. The results are expressed with reference to VPDB (Vienna Pee Dee Belemnite) as follows:

$$
\begin{aligned}
& \delta^{13} \mathrm{C}= \\
& {\left[\left(\left({ }^{13} \mathrm{C} /{ }^{12} \mathrm{C}\right)_{\text {sample }} /\left({ }^{13} \mathrm{C} /{ }^{12} \mathrm{C}\right)_{\text {standard }}\right)-1\right] \times 1000 .}
\end{aligned}
$$

The $\delta^{13} \mathrm{C}$ measurement has a precision of $0.1 \%$. A number of duplicate samples were measured, and the results show that the differences were less than the range of measurement accuracy.

\section{Results}

The major parameter and ion concentrations of samples are given in Table 1 . The $\mathrm{pH}$ values of water samples ranged from 6.50 to 8.24 , with an average of 7.23. Total dissolved solids (TDSs) of water samples varied from 35.3 to $205 \mathrm{mg} \mathrm{L}^{-1}$, with an average of $75.2 \mathrm{mg} \mathrm{L}^{-1}$. Compared with the major rivers in China, the average TDS was significantly lower than the Changjiang $\left(224 \mathrm{mg} \mathrm{L}^{-1}\right.$, Chetelat et al., 2008), the Huanghe (557 $\mathrm{mg} \mathrm{L}^{-1}$, Fan et al., 2014) and the Zhujiang (190 $\mathrm{mg} \mathrm{L}^{-1}$, S. Zhang et al., 2007). However, the average TDS was comparable to the rivers draining silicaterock-dominated areas, e.g., the upper Ganjiang in Ganzhou, south China (63 mg L ${ }^{-1}$, Ji and Jiang, 2012), the Amur in northern China (70 $\mathrm{mg} \mathrm{L}^{-1}$, Moon et al., 2009), the Xishui in Hubei, central China (101 mg L ${ }^{-1}$, Wu et al., 2013) and North Han River in South Korea (75.5 $\mathrm{mg} \mathrm{L}^{-1}$, Ryu et al., 2008). Among the major rivers in the SECRB, the Qiantang had the highest TDS value (averaging at $121 \mathrm{mg} \mathrm{L}^{-1}$ ), and the Ou had the lowest TDS value (averaging at $48.8 \mathrm{mg} \mathrm{L}^{-1}$ ).

Major ion compositions are shown in the cation and anion ternary diagrams (Fig. 2a and b). In comparison with rivers 
Table 1. Chemical and carbon isotopic compositions of river water in the Southeast Coastal River Basin (SECRB) of China.

\begin{tabular}{|c|c|c|c|c|c|c|c|c|c|c|c|c|c|c|c|c|c|c|c|c|}
\hline Rivers & $\begin{array}{l}\text { Sample } \\
\text { number }\end{array}$ & Date $^{2}$ & $\mathrm{pH}$ & $\begin{array}{r}\mathrm{T} \\
\left({ }^{\circ} \mathrm{C}\right)\end{array}$ & $\begin{array}{r}\mathrm{EC} \\
(\mu \mathrm{s} \\
\left.\mathrm{cm}^{-1}\right)\end{array}$ & $\begin{array}{l}\mathrm{Na}^{+} \\
(\mu \mathrm{M})\end{array}$ & $\begin{array}{r}\mathrm{K}^{+} \\
(\mu \mathrm{M})\end{array}$ & $\begin{array}{r}\mathrm{Mg}^{2+} \\
(\mu \mathrm{M})\end{array}$ & $\begin{array}{l}\mathrm{Ca}^{2+} \\
(\mu \mathrm{M})\end{array}$ & $\begin{array}{c}\mathrm{F}^{-} \\
(\mu \mathrm{M})\end{array}$ & $\begin{array}{c}\mathrm{Cl}^{-} \\
(\mu \mathrm{M})\end{array}$ & $\begin{array}{l}\mathrm{NO}_{3}^{-} \\
(\mu \mathrm{M})\end{array}$ & $\begin{array}{c}\mathrm{SO}_{4}^{2-} \\
(\mu \mathrm{M})\end{array}$ & $\begin{array}{r}\mathrm{HCO}_{3}^{-} \\
(\mu \mathrm{M})\end{array}$ & $\begin{array}{l}\mathrm{SiO}_{2} \\
(\mu \mathrm{M})\end{array}$ & $\begin{array}{c}\mathrm{TZ}^{+} \\
(\mu \mathrm{Eq})\end{array}$ & $\begin{array}{c}\mathrm{TZ}^{-} \\
(\mu \mathrm{Eq})\end{array}$ & $\begin{array}{r}\mathrm{NICB} \\
(\%)\end{array}$ & $\begin{array}{r}\delta^{13} \mathrm{C} \\
(\%)\end{array}$ & $\begin{array}{r}\text { TDS } \\
(\mathrm{mg} \\
\left.\mathrm{L}^{-1}\right)\end{array}$ \\
\hline \multirow[t]{18}{*}{ Qiantang $^{1}$} & 1 & 07-08-10 & 7.42 & 28.78 & 190 & 347 & 197 & 106 & 473 & 12.0 & 303 & 62.6 & 147 & 1130 & 148 & 1703 & 1789 & -5.0 & -19.0 & 144 \\
\hline & 2 & $07-09-10$ & 7.60 & 23.84 & 146 & 87.5 & 204 & 80.9 & 496 & 11.7 & 75.2 & 124 & 121 & 907 & 156 & 1446 & 1348 & 6.7 & -19.8 & 119 \\
\hline & 3 & 07-09-10 & 7.37 & 27.83 & 308 & 555 & 233 & 208 & 698 & 41.8 & 312 & 223 & 437 & 1170 & 170 & 2601 & 2579 & 0.9 & -17.8 & 204 \\
\hline & 4 & $07-10-10$ & 7.27 & 26.28 & 177 & 176 & 135 & 116 & 544 & 15.7 & 151 & 142 & 170 & 985 & 175 & 1632 & 1618 & 0.8 & -19.3 & 135 \\
\hline & 5 & $07-10-10$ & 7.05 & 24.15 & 123 & 130 & 101 & 66.2 & 349 & 17.7 & 94.3 & 124 & 157 & 529 & 169 & 1061 & 1061 & 0.0 & -18.7 & 91.2 \\
\hline & 6 & $07-10-10$ & 7.24 & 23.75 & 140 & 97.6 & 69.7 & 81.0 & 451 & 20.0 & 62.1 & 109 & 204 & 703 & 164 & 1231 & 1282 & -4.2 & -21.3 & 106.6 \\
\hline & 7 & $07-11-10$ & 7.40 & 23.23 & 107 & 92.5 & 70.5 & 68.3 & 327 & 14.9 & 74.9 & 104 & 147 & 486 & 156 & 954 & 960 & -0.6 & -21.0 & 82.2 \\
\hline & 8 & $07-11-10$ & 7.16 & 27.61 & 281 & 361 & 87.5 & 128 & 469 & 26.8 & 245 & 191 & 239 & 810 & 179 & 1642 & 1724 & -5.0 & -12.9 & 137.5 \\
\hline & 9 & $07-11-10$ & 7.02 & 26.48 & 140 & 275 & 120 & 60.7 & 319 & 36.2 & 199 & 150 & 180 & 437 & 236 & 1155 & 1146 & 0.8 & -13.9 & 100.2 \\
\hline & 10 & $07-12-10$ & 7.05 & 24.24 & 99 & 205 & 114 & 58.3 & 285 & 14.6 & 191 & 114 & 132 & 305 & 278 & 1005 & 874 & 13.1 & -20.9 & 85.4 \\
\hline & 11 & $07-12-10$ & 7.05 & 27.01 & 102 & 123 & 133 & 49.8 & 284 & 18.6 & 86.5 & 123 & 144 & 377 & 183 & 924 & 874 & 5.4 & -19.2 & 79.4 \\
\hline & 12 & $07-12-10$ & 7.99 & 24.18 & 260 & 50.0 & 85.4 & 212 & 993 & - & 66.8 & 153 & 235 & 1822 & 172 & 2546 & 2512 & 1.4 & -17.6 & 205.2 \\
\hline & 13 & $07-12-10$ & 7.86 & 24.59 & 231 & 43.5 & 88.4 & 189 & 859 & - & 55.1 & 97.6 & 169 & 1763 & 170 & 2228 & 2253 & -1.1 & -18.7 & 185.4 \\
\hline & 14 & $07-12-10$ & 7.69 & 22.66 & 131 & 44.1 & 81.0 & 113 & 458 & - & 19.1 & 95.2 & 107 & 920 & 143 & 1266 & 1248 & 1.4 & -18.1 & 106.8 \\
\hline & 15 & $07-12-10$ & 7.65 & 24.48 & 106 & 61.1 & 98.3 & 87.9 & 335 & - & 37.2 & 68.3 & 112 & 663 & 164 & 1005 & 992 & 1.4 & -18.6 & 87.3 \\
\hline & 16 & $07-12-10$ & 7.46 & 23.68 & 125 & 64.3 & 108 & 117 & 406 & - & 25.9 & 75.0 & 174 & 687 & 164 & 1218 & 1136 & 6.7 & -20.0 & 98.8 \\
\hline & 17 & $07-13-10$ & 7.33 & 24.08 & 139 & 59.8 & 116 & 136 & 429 & -29.6 & 80.4 & 209 & 752 & 162 & 1305 & 1281 & 1.9 & -20.8 & 108.1 & \\
\hline & 18 & $07-10-10$ & 7.27 & 25.74 & 141 & 163 & 114 & 69.6 & 396 & 27.3 & 126 & 148 & 161 & 597 & 153 & 1209 & 1195 & 1.1 & -21.0 & 101.0 \\
\hline \multirow[t]{4}{*}{ Cao'e } & 19 & $07-16-10$ & 7.17 & 22.27 & 108 & 212 & 86.3 & 69.4 & 183 & 5.1 & 151 & 148 & 114 & 384 & 216 & 803 & 912 & -13.5 & -21.2 & 79.1 \\
\hline & 20 & $07-16-10$ & 7.06 & 26.57 & 182 & 401 & 77.6 & 145 & 275 & 18.3 & 269 & 185 & 245 & 534 & 215 & 1318 & 1478 & -12.2 & -20.5 & 116.9 \\
\hline & 21 & $07-16-10$ & 7.14 & 27.26 & 171 & 333 & 91.3 & 164 & 362 & 18.1 & 224 & 194 & 207 & 658 & 225 & 1475 & 1490 & -1.0 & -20.9 & 123.3 \\
\hline & 22 & $07-16-10$ & 7.08 & 27.17 & 173 & 346 & 94.4 & 168 & 364 & 18.8 & 247 & 200 & 211 & 656 & 222 & 1506 & 1526 & -1.3 & -13.0 & 125.2 \\
\hline Ling & 23 & $07-15-10$ & 7.07 & 24.14 & 52 & 164 & 42.9 & 34.9 & 140 & 4.9 & 40.7 & 61.5 & 68.3 & 277 & 190 & 558 & 516 & 7.6 & -12.8 & 52.1 \\
\hline & 24 & $07-15-10$ & 7.02 & 26.04 & 74 & 169 & 92.0 & 34.2 & 150 & 6.4 & 87.0 & 77.3 & 92.8 & 272 & 196 & 629 & 622 & 1.1 & -20.8 & 59.5 \\
\hline & 25 & $07-16-10$ & 7.34 & 25.03 & 92 & 159 & 80.1 & 47.3 & 235 & 19.3 & 78.0 & 71.4 & 105 & 455 & 187 & 804 & 815 & -1.4 & -22.5 & 73.9 \\
\hline & 26 & $07-16-10$ & 7.40 & 26.75 & 113 & 216 & 77.8 & 57.1 & 249 & 20.2 & 133 & 90.0 & 115 & 494 & 196 & 905 & 946 & -4.5 & -12.7 & 82.8 \\
\hline & 27 & $07-16-10$ & 7.39 & 26 & 89 & 174 & 86.4 & 56.4 & 209 & 9.0 & 99.3 & 78.4 & 99.9 & 420 & 199 & 792 & 798 & -0.8 & -14.0 & 72.7 \\
\hline & 28 & $07-15-10$ & 6.79 & 22.33 & 75 & 159 & 82.7 & 44.1 & 143 & -107 & 61.8 & 83.4 & 306 & 144 & 616 & 641 & -4.1 & -21.1 & 56.5 & \\
\hline & 29 & $07-15-10$ & 8.24 & 27.15 & 129 & 228 & 92.1 & 83.1 & 317 & 17.2 & 177 & 90.5 & 120 & 641 & 194 & 1120 & 1148 & -2.5 & -19.2 & 97.8 \\
\hline $\mathrm{Ou}$ & 30 & $07-13-10$ & 8.08 & 28.45 & 48 & 95.2 & 107 & 38.4 & 92.1 & 15.2 & 31.8 & 43.3 & 47.4 & 291 & 221 & 463 & 461 & 0.4 & -21.7 & 50.6 \\
\hline & 31 & $07-13-10$ & 6.71 & 22.97 & 32 & 60.7 & 106 & 12.6 & 65.0 & 10.8 & 28.9 & 45.0 & 48.9 & 158 & 169 & 322 & 329 & -2.2 & -23.8 & 36.9 \\
\hline & 32 & $07-13-10$ & 7.18 & 27.59 & 73 & 107 & 127 & 36.2 & 175 & 4.3 & 57.1 & 111 & 92.0 & 283 & 210 & 655 & 634 & 3.2 & -23.4 & 62.9 \\
\hline & 33 & $07-13-10$ & 6.94 & 24.2 & 44 & 76.9 & 112 & 20.0 & 99.1 & 10.9 & 27.9 & 63.1 & 58.6 & 249 & 184 & 427 & 457 & -7.0 & -22.5 & 47.5 \\
\hline & 34 & $07-14-10$ & 7.16 & 27.45 & 90 & 187 & 127 & 41.2 & 199.5 & 17.0 & 85.6 & 102 & 116 & 367 & 251 & 796 & 787 & 1.1 & -22.4 & 76.5 \\
\hline & 35 & $07-14-10$ & 6.97 & 24.56 & 54 & 105 & 50.9 & 29.2 & 122 & 12.2 & 46.1 & 67.8 & 73.1 & 218 & 193 & 460 & 478 & -4.1 & -22.5 & 47.9 \\
\hline & 36 & $07-14-10$ & 6.82 & 21.12 & 31 & 76.4 & 133 & 12.7 & 74.5 & 7.7 & 20.7 & 36.8 & 49.1 & 192 & 162 & 383 & 348 & 9.3 & -39.5 & \\
\hline & 37 & $07-14-10$ & 6.82 & 23.69 & 45 & 89.5 & 105 & 19.0 & 97.8 & 10.6 & 39.6 & 52.8 & 59.1 & 231 & 185 & 428 & 441 & -3.0 & -22.9 & 46.2 \\
\hline & 38 & $07-15-10$ & 6.92 & 24.69 & 37 & 100 & 89.3 & 21.1 & 49.7 & 1.7 & 36.9 & 45.5 & 52.7 & 153 & 202 & 331 & 341 & -2.9 & -38.9 & \\
\hline & 39 & $07-15-10$ & 6.90 & 23.86 & 35 & 92.2 & 92.0 & 19.8 & 61.4 & 1.9 & 43.9 & 47.9 & 55.5 & 139 & 193 & 347 & 342 & 1.4 & -22.3 & 38.5 \\
\hline & 40 & $07-15-10$ & 7.09 & 25.56 & 47 & 117 & 112 & 25.7 & 83.4 & 8.0 & 52.4 & 63.1 & 57.4 & 232 & 193 & 447 & 462 & -3.3 & -22.5 & 48.1 \\
\hline & 41 & 07-14-10 & 6.97 & 24.25 & 53 & 102 & 107 & 27.6 & 119 & 13.4 & 43.5 & 59.4 & 73.2 & 277 & 183 & 502 & 526 & -4.9 & -13.7 & 52.3 \\
\hline Feiyun & 42 & $07-17-10$ & 7.28 & 25.19 & 38 & 94.0 & 81.7 & 24.0 & 75.6 & 11.4 & 59.9 & 45.7 & 51.9 & 149 & 151 & 375 & 358 & 4.5 & -37.2 & \\
\hline & 43 & $07-17-10$ & 7.08 & 25.61 & 46 & 101 & 79.9 & 33.9 & 93.4 & 4.6 & 66.2 & 55.1 & 52.8 & 223 & 151 & 435 & 450 & -3.3 & -23.7 & 43.5 \\
\hline Jiaoxi & 44 & $07-17-10$ & 7.52 & 26.92 & 47 & 116 & 81.5 & 25.2 & 92.0 & 4.1 & 73.3 & 80.3 & 25.0 & 226 & 151 & 432 & 430 & 0.5 & -23.4 & 43.0 \\
\hline & 45 & $07-17-10$ & 7.45 & 27.46 & 61 & 152 & 90.2 & 34.2 & 119 & -136 & 59.8 & 53.5 & 238 & 184 & 548 & 542 & 1.2 & -23.1 & 51.8 & \\
\hline & 46 & $07-18-10$ & 6.90 & 27.66 & 53 & 127 & 88.1 & 33.4 & 94.4 & 7.0 & 123 & 93.1 & 30.4 & 209 & 177 & 471 & 486 & -3.3 & -14.4 & 47.4 \\
\hline Huotong & 47 & $07-18-10$ & 7.34 & 24 & 43 & 116 & 78.8 & 26.1 & 58.4 & 5.4 & 68.7 & 49.7 & 20.1 & 197 & 190 & 364 & 355 & 2.3 & -22.8 & 39.6 \\
\hline Ao & 48 & $07-19-10$ & 7.24 & 31.44 & 124 & 294 & 121 & 102 & 209 & 24.3 & 204 & 73.6 & 52.0 & 717 & 370 & 1036 & 1100 & -6.1 & -19.4 & 105.4 \\
\hline & 49 & $07-19-10$ & 7.13 & 27.82 & 46 & 109 & 96.3 & 30.0 & 73.8 & -72.0 & 51.3 & 22.5 & 234 & 236 & 413 & 402 & 2.6 & -46.2 & & \\
\hline & 50 & $07-18-10$ & 6.98 & 28.65 & 53 & 140 & 88.4 & 40.8 & 100 & 3.0 & 82.9 & 58.6 & 20.9 & 294 & 233 & 511 & 477 & 6.6 & -22.3 & 52.2 \\
\hline Min & 51 & $07-27-10$ & 7.11 & 28.4 & 42 & 116 & 92.0 & 40.5 & 119 & 18.0 & 43.9 & 35.5 & 26.0 & 382 & 182 & 526 & 513 & 2.4 & -19.4 & 52.7 \\
\hline & 52 & $07-27-10$ & 7.17 & 30 & 51 & 102 & 97.9 & 41.7 & 107 & 4.6 & 29.4 & 45.3 & 35.0 & 350 & 221 & 496 & 495 & 0.2 & -53.3 & \\
\hline & 53 & $07-27-10$ & 7.08 & 29.4 & 99 & 214 & 92.7 & 46.4 & 126 & 18.4 & 50.1 & 39.8 & 118 & 327 & 154 & 651 & 654 & -0.4 & -20.8 & 74.0 \\
\hline & 54 & $07-27-10$ & 7.06 & 29.1 & 44 & 107 & 99.6 & 28.1 & 114 & 16.4 & 18.7 & 36.4 & 44.3 & 305 & 265 & 491 & 449 & 8.5 & -17.6 & 53.6 \\
\hline & 55 & $07-27-10$ & 7.42 & 29.4 & 57 & 139 & 93.7 & 49.8 & 113 & 3.1 & 67.1 & 56.3 & 26.6 & 384 & 236 & 558 & 561 & -0.5 & -16.4 & 58.6 \\
\hline & 56 & $07-27-10$ & 7.12 & 27.8 & 51 & 103 & 91.0 & 50.8 & 106 & 4.7 & 82.8 & 35.1 & 63.5 & 249 & 225 & 507 & 494 & 2.5 & -51.3 & \\
\hline & 57 & $07-27-10$ & 7.08 & 27.5 & 40 & 125 & 45.0 & 36.8 & 107 & 12.1 & 43.6 & 44.5 & 29.3 & 288 & 211 & 457 & 435 & 5.0 & -21.1 & 47.4 \\
\hline & 58 & $07-27-10$ & 6.99 & 27.2 & 52 & 121 & 98.0 & 42.4 & 115 & 16.7 & 87.1 & 36.6 & 70.9 & 277 & 228 & 535 & 542 & -1.4 & -11.4 & 55.3 \\
\hline & 59 & $07-27-10$ & 6.87 & 29 & 59 & 154 & 91.4 & 59.4 & 124 & 16.5 & 77.8 & 36.7 & 88.3 & 272 & 222 & 612 & 563 & 8.0 & -20.3 & 57.2 \\
\hline & 60 & $07-27-10$ & 7.31 & 27.1 & 78 & 109 & 92.1 & 59.1 & 181 & 21.2 & 123 & 37.5 & 78.4 & 355 & 202 & 682 & 672 & 1.4 & -18.7 & 63.1 \\
\hline & 61 & $07-27-10$ & 7.22 & 27.8 & 37 & 122 & 83.3 & 52.8 & 142 & 17.4 & 111 & 37.3 & 80.4 & 288 & 221 & 596 & 597 & -0.2 & -22.3 & 58.1 \\
\hline & 62 & $07-27-10$ & 7.16 & 28.1 & 58 & 104 & 83.3 & 59.3 & 163 & 24.0 & 34.6 & 34.5 & 118 & 294 & 214 & 632 & 599 & 5.2 & -13.4 & 59.5 \\
\hline & 63 & $07-27-10$ & 7.26 & 28.3 & 87 & 139 & 86.1 & 60.9 & 191 & 14.8 & 48.0 & 93.0 & 109 & 347 & 226 & 729 & 707 & 3.0 & -21.4 & 68.6 \\
\hline & 64 & $07-27-10$ & 7.00 & 28.8 & 87 & 127 & 93.1 & 58.7 & 195 & 6.6 & 59.8 & 81.1 & 60.9 & 480 & 232 & 729 & 743 & -2.0 & -11.0 & 74.0 \\
\hline & 65 & $07-28-10$ & 6.97 & 27.9 & 37 & 163 & 82.1 & 52.2 & 140 & 20.2 & 53.1 & 60.0 & 106 & 306 & 221 & 630 & 632 & -0.2 & -61.9 & \\
\hline & 66 & $07-13-10$ & 7.07 & 27.96 & 59 & 91.9 & 110 & 40.0 & 127 & 24.8 & 62.0 & 79.3 & 62.3 & 249 & 228 & 535 & 515 & 3.8 & -54.8 & \\
\hline & 67 & $07-28-10$ & 7.12 & 29.7 & 38 & 108 & 93.4 & 45.9 & 133 & 12.4 & 48.3 & 34.0 & 56.6 & 368 & 220 & 560 & 564 & -0.7 & -57.7 & \\
\hline & 68 & $07-27-10$ & 7.03 & 29.9 & 62 & 128 & 96.7 & 57.6 & 148 & 23.3 & 81.6 & 36.8 & 74.1 & 374 & 203 & 635 & 641 & -0.9 & -12.4 & 61.7 \\
\hline & 69 & $07-27-10$ & 7.01 & 28.8 & 60 & 102 & 89.1 & 73.6 & 138 & 9.6 & 50.6 & 74.1 & 32.7 & 417 & 233 & 615 & 607 & 1.3 & -21.0 & 62.3 \\
\hline & 70 & $07-27-10$ & 7.06 & 26.5 & 37 & 93.5 & 93.1 & 34.7 & 87.3 & -26.6 & 34.8 & 37.1 & 312 & 222 & 431 & 448 & -3.9 & -13.1 & 49.1 & \\
\hline & 71 & $07-27-10$ & 7.09 & 26.5 & 25 & 62.6 & 92.7 & 27.0 & 61.5 & 4.7 & 21.5 & 18.6 & 43.4 & 191 & 154 & 332 & 318 & 4.2 & -16.0 & 35.3 \\
\hline & 72 & $07-28-10$ & 7.07 & 30.1 & 39 & 76.3 & 87.9 & 35.1 & 87.6 & 7.4 & 43.1 & 36.6 & 35.5 & 266 & 175 & 409 & 416 & -1.7 & -19.4 & 43.5 \\
\hline
\end{tabular}


Table 1. Continued.

\begin{tabular}{|c|c|c|c|c|c|c|c|c|c|c|c|c|c|c|c|c|c|c|c|c|}
\hline Rivers & $\begin{array}{l}\text { Sample } \\
\text { number }\end{array}$ & Date $^{2}$ & $\mathrm{pH}$ & $\begin{array}{r}\mathrm{T} \\
\left({ }^{\circ} \mathrm{C}\right)\end{array}$ & $\begin{array}{r}\mathrm{EC} \\
(\mu \mathrm{s} \\
\left.\mathrm{cm}^{-1}\right)\end{array}$ & $\begin{array}{l}\mathrm{Na}^{+} \\
(\mu \mathrm{M})\end{array}$ & $\begin{array}{r}\mathrm{K}^{+} \\
(\mu \mathrm{M})\end{array}$ & $\begin{array}{r}\mathrm{Mg}^{2+} \\
(\mu \mathrm{M})\end{array}$ & $\begin{array}{l}\mathrm{Ca}^{2+} \\
(\mu \mathrm{M})\end{array}$ & $\begin{array}{c}\mathrm{F}^{-} \\
(\mu \mathrm{M})\end{array}$ & $\begin{array}{c}\mathrm{Cl}^{-} \\
(\mu \mathrm{M})\end{array}$ & $\begin{array}{l}\mathrm{NO}_{3}^{-} \\
(\mu \mathrm{M})\end{array}$ & $\begin{array}{c}\mathrm{SO}_{4}^{2-} \\
(\mu \mathrm{M})\end{array}$ & $\begin{array}{r}\mathrm{HCO}_{3}^{-} \\
(\mu \mathrm{M})\end{array}$ & $\begin{array}{l}\mathrm{SiO}_{2} \\
(\mu \mathrm{M})\end{array}$ & $\begin{array}{c}\mathrm{TZ}^{+} \\
(\mu \mathrm{Eq})\end{array}$ & $\begin{array}{c}\mathrm{TZ}^{-} \\
(\mu \mathrm{Eq})\end{array}$ & $\begin{array}{r}\text { NICB } \\
(\%)\end{array}$ & $\begin{array}{r}\delta^{13} \mathrm{C} \\
(\%)\end{array}$ & $\begin{array}{r}\text { TDS } \\
(\mathrm{mg} \\
\left.\mathrm{L}^{-1}\right)\end{array}$ \\
\hline & 73 & $07-27-10$ & 7.01 & 28.7 & 47 & 84.9 & 95.4 & 56.7 & 106 & 12.7 & 51.8 & 49.2 & 57.2 & 315 & 211 & 506 & 531 & -4.8 & -53.8 & \\
\hline & 74 & $07-27-10$ & 6.85 & 28.7 & 50 & 93.6 & 85.9 & 52.4 & 107 & 14.1 & 62.8 & 57.5 & 57.0 & 252 & 217 & 498 & 487 & 2.2 & -19.9 & 50.9 \\
\hline & 75 & $07-27-10$ & 7.11 & 29.7 & 69 & 117 & 85.2 & 73.4 & 159 & 7.6 & 63.7 & 75.2 & 47.4 & 418 & 230 & 666 & 652 & 2.2 & -22.2 & 65.0 \\
\hline & 76 & $07-28-10$ & 6.93 & 28.9 & 59 & 112 & 88.0 & 61.8 & 122 & 6.0 & 57.4 & 89.3 & 42.0 & 349 & 224 & 568 & 580 & -2.2 & -22.0 & 58.8 \\
\hline & 77 & $07-21-10$ & 7.76 & 32.4 & 51.2 & 163 & 85.5 & 52.8 & 151 & 20.2 & 55.3 & 70.3 & 78.6 & 372 & 175 & 656 & 655 & 0.3 & -12.5 & 61.8 \\
\hline & 78 & $07-28-10$ & 7.29 & 26.8 & 106 & 129 & 75.3 & 84.0 & 321 & 24.0 & 56.2 & 41.0 & 166 & 599 & 202 & 1013 & 1028 & -1.4 & -16.3 & 90.3 \\
\hline & 79 & $07-21-10$ & 7.09 & 26.96 & 56 & 112 & 87.6 & 37.1 & 129 & 4.5 & 51.5 & 44.9 & 61.9 & 327 & 276 & 531 & 547 & -2.9 & -22.2 & 59.1 \\
\hline & 80 & $07-21-10$ & 7.64 & 33.37 & 83 & 114 & 96.2 & 60.6 & 151 & 16.7 & 53.0 & 40.6 & 102 & 371 & 242 & 633 & 670 & -5.8 & -12.8 & 66.2 \\
\hline & 81 & $07-21-10$ & 7.83 & 31.27 & 65 & 131 & 102 & 52.7 & 141 & 16.1 & 45.3 & 49.7 & 91.8 & 324 & 239 & 620 & 603 & 2.8 & -13.4 & 61.8 \\
\hline & 82 & $07-21-10$ & 6.84 & 28.35 & 66 & 132 & 101 & 52.5 & 141 & 5.8 & 63.8 & 54.1 & 91.6 & 304 & 243 & 621 & 606 & 2.5 & -22.7 & 61.5 \\
\hline & 83 & $07-21-10$ & 7.42 & 30.7 & 98 & 217 & 113 & 59.2 & 210 & 18.4 & 98.7 & 63.5 & 84.7 & 496 & 320 & 868 & 827 & 4.6 & -18.9 & 84.5 \\
\hline & 84 & $07-27-10$ & 7.26 & 26.3 & 46 & 104 & 102 & 29.7 & 121 & 3.6 & 55.2 & 51.9 & 55.5 & 294 & 193 & 507 & 512 & -0.9 & -21.6 & 51.9 \\
\hline & 85 & $07-27-10$ & 7.07 & 25.4 & 30 & 73.3 & 99.2 & 19.6 & 78.8 & -22.9 & 40.0 & 49.2 & 203 & 170 & 369 & 365 & 1.3 & -21.1 & 39.8 & \\
\hline & 86 & $07-27-10$ & 7.50 & 27.3 & 45 & 102 & 102 & 26.5 & 114 & 2.4 & 35.1 & 39.7 & 57.2 & 260 & 217 & 484 & 449 & 7.3 & -15.7 & 49.6 \\
\hline & 87 & $07-27-10$ & 7.47 & 26.9 & 51 & 141 & 100 & 43.6 & 109 & 7.9 & 79.7 & 42.4 & 57.7 & 311 & 217 & 547 & 548 & -0.3 & -20.1 & 55.6 \\
\hline & 88 & $07-19-10$ & 7.99 & 31.74 & 63 & 167 & 96.5 & 33.5 & 115 & 8.0 & 105 & 35.5 & 38.1 & 331 & 218 & 561 & 548 & 2.3 & -13.5 & 55.9 \\
\hline & 89 & $07-21-10$ & 6.77 & 28.19 & 65 & 132 & 93.6 & 56.0 & 145 & 15.6 & 60.6 & 78.8 & 75.4 & 333 & 243 & 627 & 624 & 0.5 & -22.6 & 63.3 \\
\hline \multirow[t]{4}{*}{ Jin } & 90 & $07-27-10$ & 7.36 & 25.8 & 128 & 126 & 94.8 & 88.9 & 406 & 22.9 & 51.4 & 39.4 & 229 & 595 & 208 & 1211 & 1143 & 5.6 & -20.7 & 100 \\
\hline & 91 & $07-27-10$ & 7.40 & 26.9 & 123 & 143 & 103 & 82.7 & 347 & 21.0 & 83.5 & 203 & 182 & 463 & 226 & 1105 & 1115 & -0.9 & -21.3 & 98.4 \\
\hline & 92 & $07-27-10$ & 7.00 & 27.4 & 88 & 170 & 98.8 & 56.8 & 205 & 7.2 & 137 & 117 & 106 & 327 & 205 & 793 & 792 & 0.1 & -22.5 & 71.8 \\
\hline & 93 & $07-27-10$ & 7.32 & 28.7 & 73 & 201 & 116 & 87.1 & 318 & 20.0 & 93.5 & 41.5 & 189 & 508 & 267 & 1128 & 1020 & 9.6 & -21.7 & 95.3 \\
\hline \multirow[t]{11}{*}{ Jiulong } & 94 & $07-30-10$ & 6.50 & 23.47 & 29 & 72.3 & 92.4 & 22.8 & 59.8 & 12.4 & 25.1 & 27.0 & 50.0 & 189 & 213 & 330 & 341 & -3.4 & -18.1 & 40.1 \\
\hline & 95 & $07-30-10$ & 7.06 & 29.35 & 120 & 136 & 96.9 & 106 & 339 & 5.1 & 67.7 & 66.3 & 249 & 469 & 202 & 1124 & 1100 & 2.1 & -20.8 & 94.2 \\
\hline & 96 & $07-30-10$ & 7.45 & 27.6 & 104 & 79.5 & 97.5 & 106 & 363 & 14.4 & 70.7 & 50.0 & 99.9 & 729 & 184 & 1116 & 1049 & 6.0 & -18.9 & 93.7 \\
\hline & 97 & $07-31-10$ & 7.36 & 26.59 & 139 & 140 & 100 & 142 & 432 & 15.5 & 79.6 & 78.3 & 274 & 573 & 196 & 1388 & 1278 & 8.0 & -19.7 & 108.8 \\
\hline & 98 & $07-31-10$ & 7.72 & 26.18 & 88 & 77.6 & 96.2 & 69.0 & 313 & 19.9 & 39.7 & 34.6 & 63.8 & 731 & 251 & 938 & 933 & 0.5 & -18.4 & 89.4 \\
\hline & 99 & $07-30-10$ & 7.43 & 26.96 & 119 & 200 & 93.8 & 100.2 & 298 & 19.9 & 122 & 80.5 & 225 & 387 & 202 & 1091 & 1040 & 4.7 & -20.5 & 89.5 \\
\hline & 100 & $07-28-10$ & 7.41 & 26.66 & 112 & 173 & 97.9 & 94.4 & 286 & 46.1 & 118 & 152 & 201 & 364 & 207 & 1033 & 1036 & -0.3 & -20.9 & 92.2 \\
\hline & 101 & $07-29-10$ & 7.16 & 29.35 & 82 & 151 & 110 & 55.4 & 178 & 4.9 & 71.2 & 170 & 53.2 & 385 & 305 & 727 & 732 & -0.7 & -21.2 & 76.1 \\
\hline & 102 & $07-29-10$ & 7.10 & 28.9 & 100 & 222 & 98.3 & 49.4 & 249 & 3.6 & 126 & 157 & 52.7 & 532 & 303 & 917 & 920 & -0.3 & -21.7 & 90.0 \\
\hline & 103 & $07-28-10$ & 7.20 & 31.15 & 138 & 339 & 111 & 81.2 & 277 & 9.2 & 280 & 285 & 88.6 & 515 & 317 & 1165 & 1256 & -7.8 & -19.0 & 112 \\
\hline & 104 & $07-28-10$ & 7.16 & 27.09 & 101 & 261 & 95.8 & 81.7 & 235 & 40.3 & 173 & 80.1 & 174 & 291 & 136 & 990 & 892 & 9.9 & -24.3 & 75.4 \\
\hline Zhang & 105 & $07-28-10$ & 8.08 & 30.6 & 93 & 195 & 96.1 & 61.1 & 167 & 16.8 & 157 & 193 & 55.2 & 281 & 288 & 748 & 741 & 0.9 & -21.5 & 73.8 \\
\hline Dongxi & 106 & $07-28-10$ & 7.20 & 30.9 & 78 & 263 & 99.0 & 41.5 & 115 & 14.5 & 238 & 65.3 & 30.0 & 283 & 309 & 675 & 646 & 4.4 & -20.8 & 66.7 \\
\hline Huangang & 107 & $07-28-10$ & 7.40 & 30.5 & 99 & 253 & 85.6 & 53.0 & 154 & 7.7 & 190 & 63.5 & 56.4 & 460 & 278 & 754 & 827 & -9.6 & -20.0 & 77.4 \\
\hline \multirow[t]{12}{*}{ Han } & 108 & $07-31-10$ & 7.31 & 27.1 & 68 & 136 & 61.5 & 45.2 & 195 & 16.1 & 37.7 & 45.3 & 93.7 & 345 & 218 & 678 & 615 & 9.2 & -21.9 & 62.0 \\
\hline & 109 & $07-30-10$ & 7.38 & 26.94 & 88 & 116 & 103 & 63.6 & 265 & 6.4 & 53.4 & 72.2 & 84.9 & 584 & 244 & 876 & 879 & -0.4 & -20.4 & 83.7 \\
\hline & 110 & $07-30-10$ & 6.66 & 25.55 & 71 & 114 & 96.2 & 47.6 & 168 & 8.0 & 56.9 & 54.6 & 143 & 230 & 203 & 642 & 628 & 2.2 & -17.9 & 59.7 \\
\hline & 111 & $07-30-10$ & 6.66 & 27.76 & 83 & 135 & 104 & 63.8 & 203 & 8.6 & 54.5 & 74.9 & 173 & 302 & 336 & 774 & 777 & -0.4 & -20.6 & 78.7 \\
\hline & 112 & $07-30-10$ & 7.31 & 30.81 & 56 & 168 & 74.0 & 39.1 & 118 & 13.5 & 62.9 & 44.4 & 81.4 & 237 & 245 & 556 & 507 & 8.8 & -21.4 & 54.6 \\
\hline & 113 & 07-31-10 & 7.28 & 28.73 & 98 & 137 & 99.3 & 85.6 & 270 & 9.2 & 88.8 & 59.1 & 118 & 565 & 233 & 948 & 949 & -0.1 & -19.7 & 86.6 \\
\hline & 114 & $07-31-10$ & 7.27 & 31.42 & 123 & 193 & 105 & 98.2 & 319 & 20.7 & 120 & 102 & 157 & 570 & 229 & 1132 & 1107 & 2.2 & -19.7 & 98.2 \\
\hline & 115 & $07-30-10$ & 7.43 & 29.89 & 85 & 115 & 97.5 & 65.5 & 244 & 6.5 & 46.5 & 58.6 & 103 & 511 & 251 & 832 & 822 & 1.1 & -20.8 & 79.3 \\
\hline & 116 & $07-31-10$ & 7.61 & 30.98 & 99 & 123 & 104 & 85.9 & 264 & 5.6 & 58.8 & 90.9 & 108 & 588 & 98 & 926 & 952 & -2.9 & -20.0 & 79.4 \\
\hline & 117 & $07-31-10$ & 7.31 & 29.96 & 93 & 151 & 103 & 78.1 & 250 & 15.4 & 68.0 & 99.1 & 173 & 379 & 233 & 909 & 891 & 1.9 & -21.9 & 81.8 \\
\hline & 118 & $07-31-10$ & 7.35 & 28.4 & 2 & 233 & 84.2 & 101 & 323 & 12.8 & 84.0 & 101 & 203 & 460 & 229 & 1165 & 1051 & 9.8 & -21.1 & 94.7 \\
\hline & 119 & $07-31-10$ & 7.67 & 30.38 & 93 & 136 & 87.8 & 73.6 & 231 & 16.4 & 64.6 & 94.4 & 184 & 382 & 226 & 834 & 909 & -9.1 & -20.8 & 80.5 \\
\hline \multirow[t]{2}{*}{ Rong } & 120 & $07-30-10$ & 7.57 & 31.83 & 68 & 193 & 79.1 & 50.3 & 146 & 16.4 & 192 & 84.0 & 31.5 & 344 & 309 & 664 & 683 & -2.8 & -20.3 & 65.8 \\
\hline & 121 & $07-30-10$ & 6.96 & 30.62 & 94 & 509 & 103 & 56.1 & 213 & 15.9 & 511 & 78.5 & 82.3 & 379 & 222 & 1150 & 1133 & 1.5 & -20.0 & 94.4 \\
\hline
\end{tabular}

$\mathrm{TZ}^{+}$is the total cationic charge, $\mathrm{TZ}^{-}$is the total anionic charge, NICB is the normalized inorganic charge balance and TDS is the total dissolved solid.

${ }^{1}$ Data on major ion compositions are from L. Liu et al. (2016). ${ }^{2}$ Date format is $\mathrm{mm} / \mathrm{dd} / \mathrm{yy}$.

(e.g., the Wujiang and Xijiang) draining carbonate-rockdominated areas (Han and Liu, 2004; Xu and Liu, 2010), these rivers in the SECRB had distinctly higher proportions of $\mathrm{Na}^{+}, \mathrm{K}^{+}$and dissolved $\mathrm{SiO}_{2}$. As shown in the Fig. 2, most samples had high $\mathrm{Na}^{+}$and $\mathrm{K}^{+}$proportions, with an average of more than $50 \%$ (in $\mu \mathrm{mol} \mathrm{L}^{-1}$ ) of the total cations, except for samples from the Qiantang. The concentrations of $\mathrm{Na}^{+}$and $\mathrm{K}^{+}$ranged from 43.5 to $555 \mu \mathrm{mol} \mathrm{L}^{-1}$ and 42.9 to $233 \mu \mathrm{mol} \mathrm{L}-1$, with average values of 152 and $98 \mu \mathrm{mol} \mathrm{L}^{-1}$, respectively. The concentrations of dissolved $\mathrm{SiO}_{2}$ ranged from 98.5 to $370 \mu \mathrm{mol} \mathrm{L}^{-1}$, with an average of $212 \mu \mathrm{mol} \mathrm{L}^{-1}$. $\mathrm{Ca}^{2+}$ and $\mathrm{Mg}^{2+}$ accounted for about $38 \%$ and $11.6 \%$ of the total cation concentrations. $\mathrm{HCO}_{3}^{-}$was the dominant anion, with concentrations ranging from 139 to $1822 \mu \mathrm{mol} \mathrm{L}^{-1}$.
On average, it comprised $60.6 \%$ (36\%-84.6\%) of total anions on a molar unit basis, followed by $\mathrm{SO}_{4}^{2-}(14.6 \%), \mathrm{Cl}^{-}$ $(13.1 \%)$ and $\mathrm{NO}_{3}^{-}(11.8 \%)$. The major ionic compositions indicate that the water chemistry of these rivers in the SECRB is controlled by silicate weathering. Meanwhile, it is also influenced by carbonate weathering, especially for the Qiantang catchment.

The $\delta^{13} \mathrm{C}$ of dissolved inorganic carbon in the rivers of the SECRB are also given in Table 1 . The $\delta^{13} \mathrm{C}$ of the water samples showed a wide range, from $-11.0 \%$ to $-24.3 \%$ (averaging at $-19.4 \% o$ ), and with a majority of samples falling into the range of $-15 \%$ o to $-23 \%$. The values are comparable to rivers draining the Deccan Traps (Das et al., 2005). 

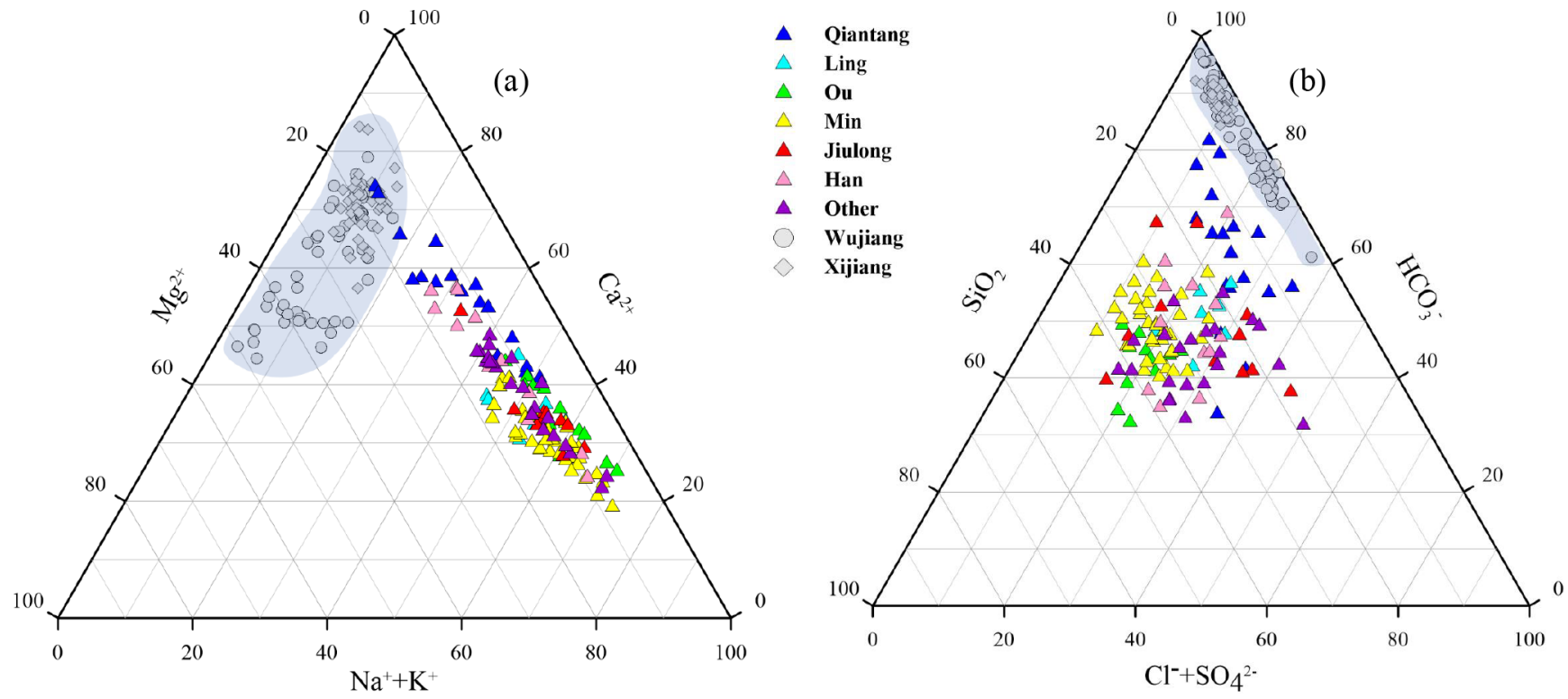

Figure 2. Ternary diagrams showing (a) cations, (b) anions and dissolved $\mathrm{SiO}_{2}$ compositions of river water in the $\mathrm{SECRB}$. Chemical compositions from case studies of rivers draining carbonate rocks (the Wujiang and the Xijiang) are also shown for comparison (data from Han and Liu, 2004; Xu and Liu 2007, 2010).

\section{Discussion}

The dissolved solids in river water are commonly from atmospheric and anthropogenic inputs and weathering of rocks within the drainage basin. It is necessary to quantify the contribution of different sources to the dissolved loads before deriving chemical weathering rates and associated $\mathrm{CO}_{2}$ consumption.

\subsection{Atmospheric and anthropogenic inputs}

To evaluate atmospheric inputs to river water, chloride is the most commonly used reference. Generally, water samples that have the lowest $\mathrm{Cl}^{-}$concentrations are employed to correct the proportion of atmospheric inputs in a river system (Négrel et al., 1993; Gaillardet et al., 1997; Viers et al., 2001; Xu and Liu, 2007). In pristine areas, the concentration of $\mathrm{Cl}^{-}$in river water is assumed to be entirely derived from the atmosphere, provided that the contribution of evaporites is negligible (e.g., Stallard and Edmond, 1981; Négrel et al., 1993). In the SECRB, the lowest $\mathrm{Cl}^{-}$concentration was mainly found in the headwater of each river. According to the geologic setting, no salt-bearing rocks were found in these headwater areas (FJBGRM, 1985; ZJBGMR, 1989). In addition, these areas are mainly mountainous and sparsely populated. Therefore, we assumed that the lowest $\mathrm{Cl}^{-}$concentration of samples from the headwater of each major river came entirely from the atmosphere.

The proportion of atmosphere-derived ions in river water can then be calculated by the element / $\mathrm{Cl}$ ratios of the rain. Chemical compositions of rain in the studied area have been reported at different sites, including Hangzhou, Jinhua, Nanping, Fuzhou and Xiamen (Zhao, 2004; M. Zhang et al., 2007; Huang et al., 2008; Cheng et al., 2011; Xu et al., 2011) (Fig. 1). The volume-weighted mean concentration of ions and $\mathrm{Cl}$-normalized molar ratios are compiled in Table 2. Based on this procedure, $6.6 \%-23.4 \%$ (averaging 14.3\%) of total dissolved cations in the major rivers of the SECRB originated from rain. Among the anions, $\mathrm{SO}_{4}^{2-}$ and $\mathrm{NO}_{3}^{-}$in the rivers are mainly from the atmospheric input, averaging $73.2 \%$ for $\mathrm{SO}_{4}^{2-}$ and $75.8 \%$ for $\mathrm{NO}_{3}^{-}$, respectively.

As one of the most developed and populated areas in China, the chemistry of river water in the SECRB could be significantly impacted by anthropogenic inputs. $\mathrm{Cl}^{-}, \mathrm{NO}_{3}^{-}$ and $\mathrm{SO}_{4}^{2-}$ are commonly associated with anthropogenic sources and have been used as tracers of anthropogenic inputs in watershed. High concentrations of $\mathrm{Cl}^{-}, \mathrm{NO}_{3}^{-}$and $\mathrm{SO}_{4}^{2-}$ can be found in the lower reaches of rivers in the SE$\mathrm{CRB}$, and there is an obvious increase after it had flowed through plains and cities. This tendency indicates that the river water chemistry is affected by anthropogenic inputs while passing through the catchments. After correcting for the atmospheric contribution to river water, the following assumption is needed to quantitatively estimate the contributions of anthropogenic inputs, which is that $\mathrm{Cl}^{-}$originates from only atmospheric and anthropogenic inputs, and the excess of atmospheric $\mathrm{Cl}^{-}$is regarded to present anthropogenic inputs and is balanced by $\mathrm{Na}^{+}$. 


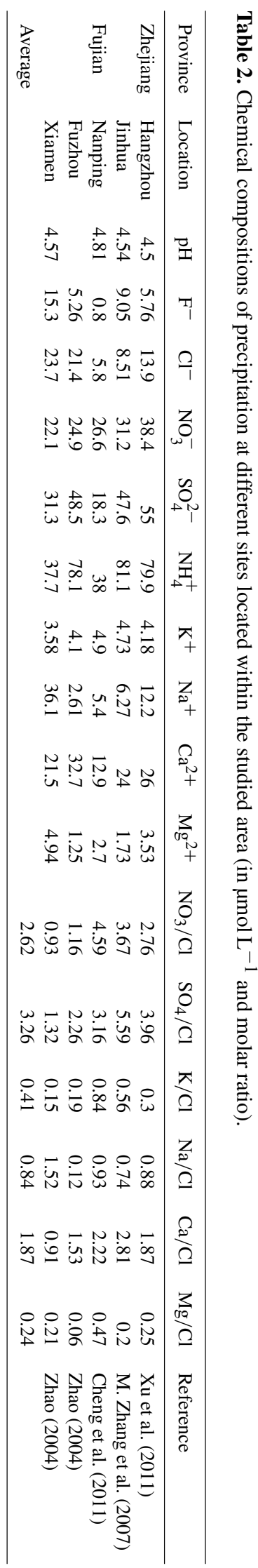

\subsection{Chemical weathering inputs}

Water samples were plotted with Na-normalized molar ratios (Fig. 3). The values of the world's major rivers (Gaillardet et al., 1999) are also shown in the figure. The best correlations between elemental ratios were observed for $\mathrm{Ca}^{2+} / \mathrm{Na}^{+}$ vs. $\mathrm{Mg}^{2+} / \mathrm{Na}^{+}\left(R^{2}=0.95, n=120\right)$ and $\mathrm{Ca}^{2+} / \mathrm{Na}^{+}$vs. $\mathrm{HCO}_{3}^{-} / \mathrm{Na}^{+}\left(R^{2}=0.98, n=120\right)$. The samples cluster on a mixing line, mainly between silicate and carbonate endmembers, closer to the silicate end-member and show little evaporite contribution. This corresponds with the rock type distributions in the SECRB. In addition, all water samples have equivalent ratios of $\left(\mathrm{Na}^{+}+\mathrm{K}^{+}\right) / \mathrm{Cl}^{-}$larger than one, indicating silicate weathering as the source of $\mathrm{Na}^{+}$and $\mathrm{K}^{+}$ rather than dissolution of chloride evaporites.

The geochemical characteristics of the silicate and carbonate end-members can be deduced from the correlations between elemental ratios and referred to literature data for catchments with well-constrained lithology. After correction for atmospheric inputs, the $\mathrm{Ca}^{2+} / \mathrm{Na}^{+}, \mathrm{Mg}^{2+} / \mathrm{Na}^{+}$and $\mathrm{HCO}_{3}^{-} / \mathrm{Na}^{+}$of the river samples ranged from 0.31 to 30 , 0.16 to 6.7 and 1.1 to 64.2 , respectively. According to the geological setting (Fig. 1), there are some small rivers draining purely silicate areas in the SECR drainage basins. Based on the elemental ratios of these rivers, we assigned the silicate end-member for this study to $\mathrm{Ca}^{2+} / \mathrm{Na}^{+}=0.41 \pm 0.10$, $\mathrm{Mg}^{2+} / \mathrm{Na}^{+}=0.20 \pm 0.03$ and $\mathrm{HCO}_{3}^{-} / \mathrm{Na}^{+}=1.7 \pm 0.6$. The ratio of $\left(\mathrm{Ca}^{2+}+\mathrm{Mg}^{2+}\right) / \mathrm{Na}^{+}$for the silicate end-member was $0.61 \pm 0.13$, which is close to the silicate end-member for the world's rivers $\left(\left(\mathrm{Ca}^{2+}+\mathrm{Mg}^{2+}\right) / \mathrm{Na}^{+}=0.59 \pm 0.17\right.$, Gaillardet et al., 1999). Moreover, previous research has documented the chemical composition of rivers, such as the Amur and the Songhuajiang in northern China, the Xishui in the lower reaches of the Changjiang, and major rivers in South Korea (Moon et al., 2009; Liu et al., 2013; Wu et al., 2013; Ryu et al., 2008; Shin et al., 2011). These river basins have similar lithological settings to the study area, so we could further validate the composition of the silicate end-member with their results. $\mathrm{Ca}^{2+} / \mathrm{Na}^{+}$and $\mathrm{Mg}^{2+} / \mathrm{Na}^{+}$ratios of silicate end-member were reported for the Amur (0.36 and 0.22), the Songhuajiang $(0.44 \pm 0.23$ and 0.16$)$, the Xishui $(0.6 \pm 0.4$ and $0.32 \pm 0.18)$, the Han $(0.55$ and 0.21$)$ and six major rivers in South Korea $(0.48$ and 0.20$)$ in the studies above, well bracketing our estimation for the silicate end-member.

However, some samples show high concentrations of $\mathrm{Ca}^{2+}, \mathrm{Mg}^{2+}$ and $\mathrm{HCO}_{3}^{-}$, indicating the contribution of carbonate weathering. The samples from the upper reaches (Sample 12 and 13) of the Qiantang fall close to the carbonate end-member documented for the world's major rivers (Gaillardet et al., 1999). In the present study, the $\mathrm{Ca}^{2+} / \mathrm{Na}^{+}$ ratio of $0.41 \pm 0.10$ and $\mathrm{Mg}^{2+} / \mathrm{Na}^{+}$ratio of $0.20 \pm 0.03$ for the silicate end-member are used to calculate the contribution of $\mathrm{Ca}^{2+}$ and $\mathrm{Mg}^{2+}$ from silicate weathering. Finally, residual $\mathrm{Ca}^{2+}$ and $\mathrm{Mg}^{2+}$ are attributed to carbonate weathering. 

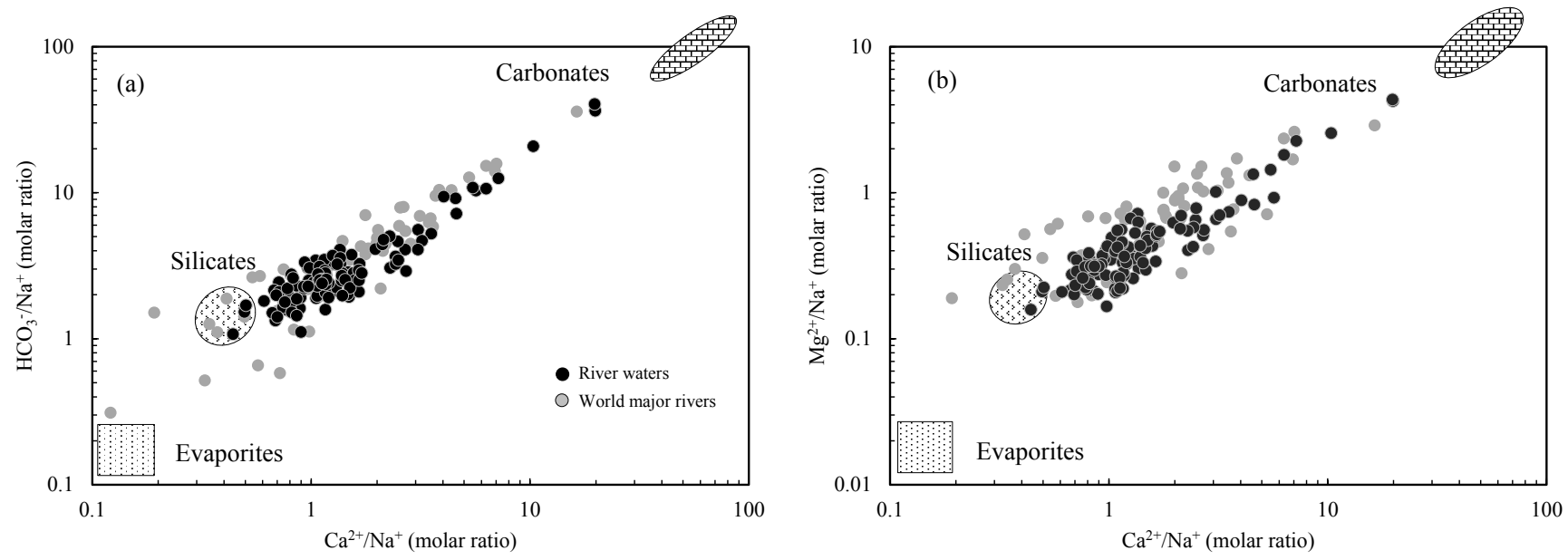

Figure 3. Mixing diagrams using Na-normalized molar ratios: (a) $\mathrm{HCO}_{3}^{-} / \mathrm{Na}^{+}$vs. $\mathrm{Ca}^{2+} / \mathrm{Na}^{+}$and (b) $\mathrm{Mg}^{2+} / \mathrm{Na}^{+}$vs. $\mathrm{Ca}^{2+} / \mathrm{Na}^{+}$for the SECRB. The samples mainly cluster on a mixing line between silicate and carbonate end-members. Data for the world's major rivers are also plotted (data from Gaillardet et al., 1999).

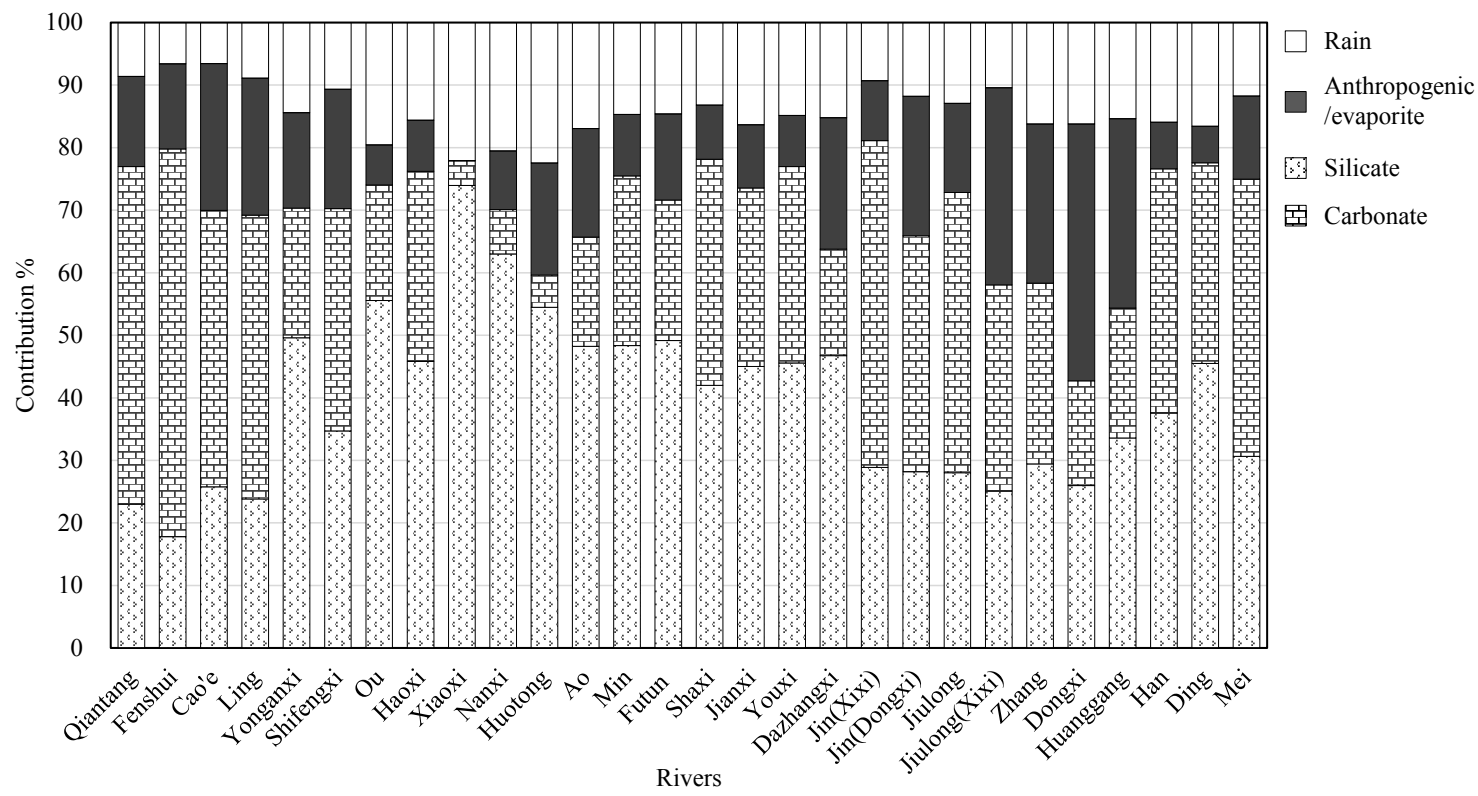

Figure 4. Calculated contributions (in \%) from the different reservoirs to the total cationic load for major rivers and their main tributaries in the SECRB. The cationic loads are the sum of $\mathrm{Na}^{+}, \mathrm{K}^{+}, \mathrm{Ca}^{2+}$ and $\mathrm{Mg}^{2+}$ from different reservoirs.

\subsection{Chemical weathering rate in the SECRB}

Based on the above assumption, a forward model is employed to quantify the relative contribution of the different sources to the rivers of the SECRB in this study (e.g., Galy and France-Lanord, 1999; Moon et al., 2007; Xu and Liu, 2007, 2010; Liu et al., 2013). The calculated contributions of different reservoirs to the total cationic load for major rivers and their main tributaries in the SECRB are presented in Fig. 4. On average, the dissolved cationic loads of the rivers in the study area originate dominantly from sili- cate weathering, which accounts for $39.5 \%(17.8 \%-74.0 \%)$ of the total cationic loads in molar unit. Carbonate weathering and anthropogenic inputs account for $30.6 \%$ (3.9\%$62.0 \%)$ and $15.7 \%(0 \%-41.1 \%)$, respectively. Contributions from silicate weathering are high in the $\mathrm{Ou}(55.6 \%)$, the Huotong $(54.5 \%)$, the Ao $(48.3 \%)$ and the Min $(48.3 \%)$ river catchments, which are dominated by granitic and volcanic bedrock. In contrast, a high contribution from carbonate weathering is observed in the Qiantang (54.0\%), the Jin $(52.2 \%)$ and the Jiulong $(44.8 \%)$ river catchments. 
The results manifest the lithology control on river solutes of drainage basin.

The chemical weathering rate of rocks is estimated by the mass budget, basin area and annual discharge (data from Hydrological data of river basins in Zhejiang, Fujian province and Taiwan region, Annual Hydrological Report of 2010, P. R. China, Table 3), expressed in $\mathrm{t} \mathrm{km}^{-2} \mathrm{a}^{-1}$. The silicate weathering rate (SWR) is calculated using major cationic concentrations from silicate weathering and assuming that all dissolved $\mathrm{SiO}_{2}$ is derived from silicate weathering ( $\mathrm{Xu}$ and Liu, 2010), as in the equation below:

$$
\begin{aligned}
\mathrm{SWR} & =\left([\mathrm{Na}]_{\text {sil }}+[\mathrm{K}]_{\text {sil }}+[\mathrm{Ca}]_{\text {sil }}+[\mathrm{Mg}]_{\text {sil }}\right. \\
& \left.+\left[\mathrm{SiO}_{2}\right]_{\text {riv }}\right) \times \text { discharge } / \text { area }
\end{aligned}
$$

The assumption about Si could lead to an overestimation of the silicate weathering rate, as part of the silica may come from dissolution of biogenic materials rather than the weathering of silicate minerals (Millot et al., 2003; Shin et al., 2011). Thus, the cationic silicate weathering rates $\left(\mathrm{Cat}_{\mathrm{sil}}\right)$ were also calculated.

The carbonate weathering rate (CWR) is calculated based on the sum of $\mathrm{Ca}^{2+}, \mathrm{Mg}^{2+}$ and $\mathrm{HCO}_{3}^{-}$from carbonate weathering, with half of the $\mathrm{HCO}_{3}^{-}$coming from carbonate weathering and being derived from the atmosphere $\mathrm{CO}_{2}$, as in the equation below:

$\mathrm{CWR}=$

$\left([\mathrm{Ca}]_{\text {carb }}+[\mathrm{Mg}]_{\text {carb }}+1 / 2\left[\mathrm{HCO}_{3}\right]_{\text {carb }}\right) \times$ discharge/area

The chemical weathering rate and flux are calculated for major rivers and their main tributaries in the SECRB, and the results are shown in Table 3. Silicate and carbonate weathering fluxes of these rivers (SWF and CWF) range from $0.02 \times 10^{6}$ to $1.80 \times 10^{6} \mathrm{ta}^{-1}$ and from $0.004 \times 10^{6}$ to $1.74 \times 10^{6} \mathrm{ta}^{-1}$, respectively. Out of the rivers, the Min has the highest silicate weathering flux, and the Qiantang has the highest carbonate weathering flux. On the whole SECRB scale, $3.95 \times 10^{6}$ and $4.09 \times 10^{6} \mathrm{ta}^{-1}$ of dissolved solids originating from silicate and carbonate weathering, respectively, are transported into the East and South China seas by rivers in this region. Compared with the largest three river basins (the Changiiang, the Huanghe and the Xijiang) in China, the flux of silicate weathering calculated for the SECRB is lower than the Changjiang $\left(9.5 \times 10^{6} \mathrm{ta}^{-1}\right.$, Gaillardet et al., 1999) but higher than the Huanghe $\left(1.52 \times 10^{6} \mathrm{ta}^{-1}\right.$, Fan et al., 2014) and the Xijiang $\left(2.62 \times 10^{6} \mathrm{ta}^{-1}\right.$, Xu and Liu, 2010).

The silicate and carbonate chemical weathering rates for these river watersheds were 14.2-35.8 and 1.8$52.1 \mathrm{t} \mathrm{km}^{-2} \mathrm{a}^{-1}$, respectively. The total rock weathering rate (TWR) for the whole SECRB is $48.1 \mathrm{t} \mathrm{km}^{-2} \mathrm{a}^{-1}$, higher than the world average $\left(24 \mathrm{t} \mathrm{km}^{-2} \mathrm{a}^{-1}\right.$, Gaillardet et al., 1999). The cationic silicate weathering rates $\left(\mathrm{Cat}_{\text {sil }}\right)$ range from 4.7 to $12.0 \mathrm{t} \mathrm{km}^{-2} \mathrm{a}^{-1}$ for the river watersheds in the SECRB, averaging at $7.8 \mathrm{t} \mathrm{km}^{-2} \mathrm{a}^{-1}$. Furthermore, a good linear correlation $\left(R^{2}=0.77, n=28\right)$ is observed between the Cat ${ }_{\text {sil }}$

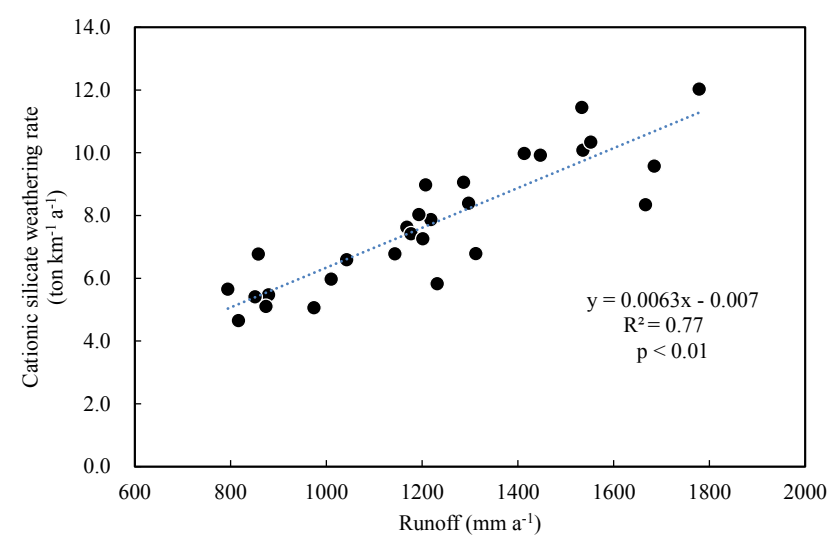

Figure 5. Plots of the cationic-silicate weathering rate $\left(\mathrm{Cat}_{\text {sil }}\right)$ vs. runoff for the SECRB.

and runoff (Fig. 5), indicating that silicate weathering rates are controlled by runoff as documented in previous research (e.g., Bluth and Kump, 1994; Gaillardet et al., 1999; Millot et al., 2002; Oliva et al., 2003; Wu et al., 2013; Pepin et al., 2013).

\section{$5.4 \mathrm{CO}_{2}$ consumption and the role of sulfuric acid}

To calculate atmospheric $\mathrm{CO}_{2}$ consumption by silicate weathering (CSW) and by carbonate weathering $(\mathrm{CCW})$, a charge-balanced state between rock chemical weatheringderived alkalinity and cations was assumed (Roy et al., 1999).

$$
\begin{aligned}
& {\left[\mathrm{CO}_{2}\right]_{\mathrm{CSW}}=} \\
& {\left[\mathrm{HCO}_{3}\right]_{\mathrm{CSW}}=[\mathrm{Na}]_{\mathrm{sil}}+[\mathrm{K}]_{\text {sil }}+2[\mathrm{Ca}]_{\mathrm{sil}}+2[\mathrm{Mg}]_{\mathrm{sil}}} \\
& {\left[\mathrm{CO}_{2}\right]_{\mathrm{CCW}}=[\mathrm{HCO} 3]_{\mathrm{CCW}}=[\mathrm{Ca}]_{\text {carb }}+[\mathrm{Mg}]_{\text {carb }}}
\end{aligned}
$$

The calculated $\mathrm{CO}_{2}$ consumption rates by chemical weathering for the rivers in SECRB are shown in Table 3. $\mathrm{CO}_{2}$ consumption rates by carbonate and silicate weathering are from 17.9 to $530 \times 10^{3} \mathrm{~mol} \mathrm{~km}^{-2} \mathrm{a}^{-1}$ (averaging at $\left.206 \times 10^{3} \mathrm{~mol} \mathrm{~km}^{-2} \mathrm{a}^{-1}\right)$ and from 167 to $460 \times$ $10^{3} \mathrm{~mol} \mathrm{~km}^{-2} \mathrm{a}^{-1}$ (averaging at $281 \times 10^{3} \mathrm{~mol} \mathrm{~km}^{-2} \mathrm{a}^{-1}$ ) for major river catchments in the SECRB. The $\mathrm{CO}_{2}$ consumption rates by silicate weathering in the SECRB are higher than that of major rivers in the world and China, such as the Amazon $\left(174 \times 10^{3} \mathrm{~mol} \mathrm{~km}^{-2} \mathrm{a}^{-1}\right.$, Mortatti and Probst, 2003), the Mississippi and the Mackenzie (66.8 and $34.1 \times$ $10^{3} \mathrm{~mol} \mathrm{~km}^{-2} \mathrm{a}^{-1}$, Gaillardet et al., 1999), the Changjiang $\left(112 \times 10^{3} \mathrm{~mol} \mathrm{~km}^{-2} \mathrm{a}^{-1}\right.$, Chetelat et al., 2008), the Huanghe $\left(35 \times 10^{3} \mathrm{~mol} \mathrm{~km}^{-2} \mathrm{a}^{-1}\right.$, Fan et al., 2014), the Xijiang (154× $10^{3} \mathrm{~mol} \mathrm{~km}^{-2} \mathrm{a}^{-1}$, Xu and Liu, 2010), the Longchuanjiang $\left(173 \times 10^{3} \mathrm{~mol} \mathrm{~km}^{-2} \mathrm{a}^{-1}\right.$, Li et al., 2011), the Mekong (191× $10^{3} \mathrm{~mol} \mathrm{~km}^{-2} \mathrm{a}^{-1}$, Li et al., 2014), three large rivers in eastern Tibet (103-121 × $10^{3} \mathrm{~mol} \mathrm{~km}^{-2} \mathrm{a}^{-1}$, Noh et al., 2009), the Hanjiang in central China $\left(120 \times 10^{3} \mathrm{~mol} \mathrm{~km}^{-2} \mathrm{a}^{-1}\right.$, Li et al., 2009) and the Sonhuajiang in northeastern China 


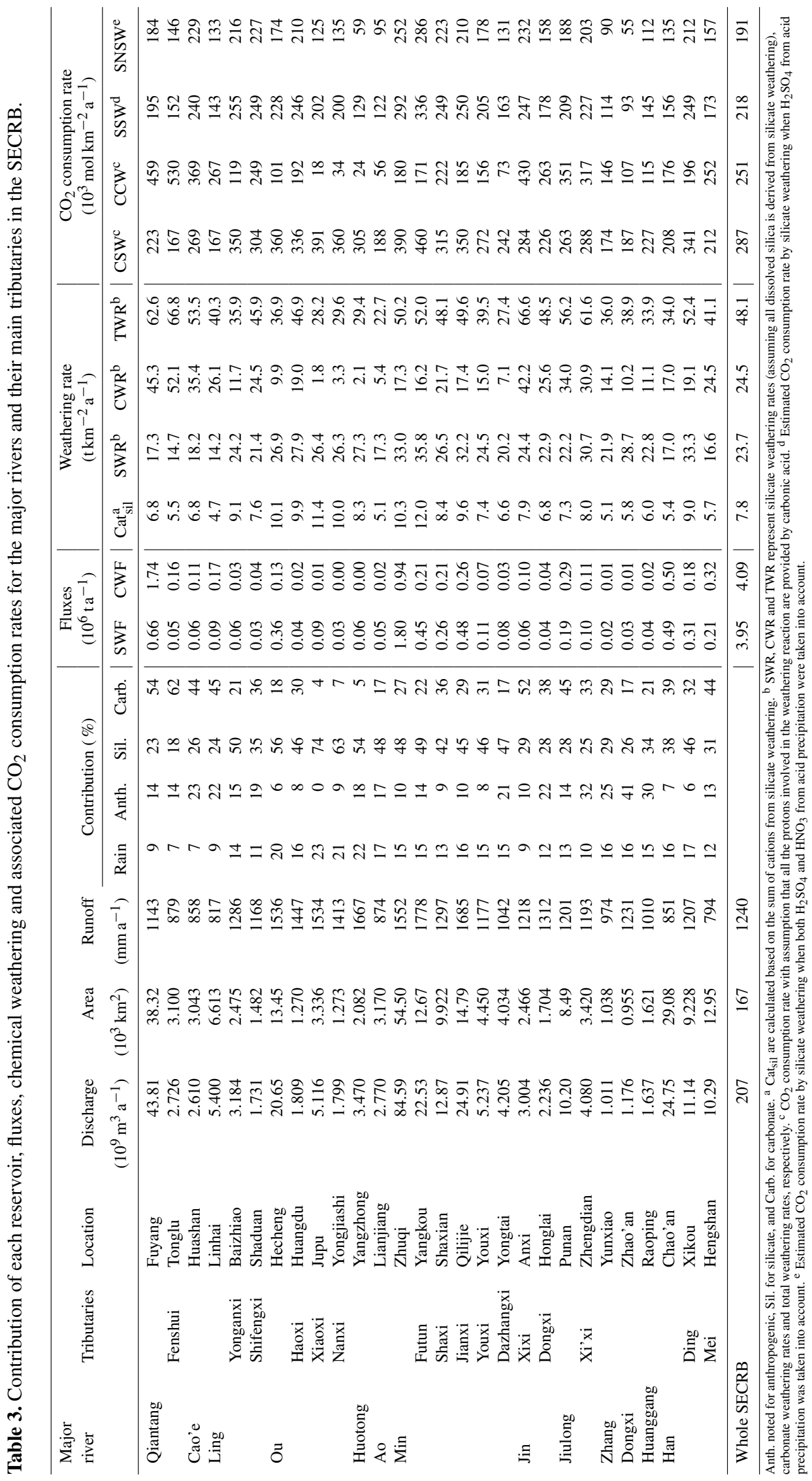



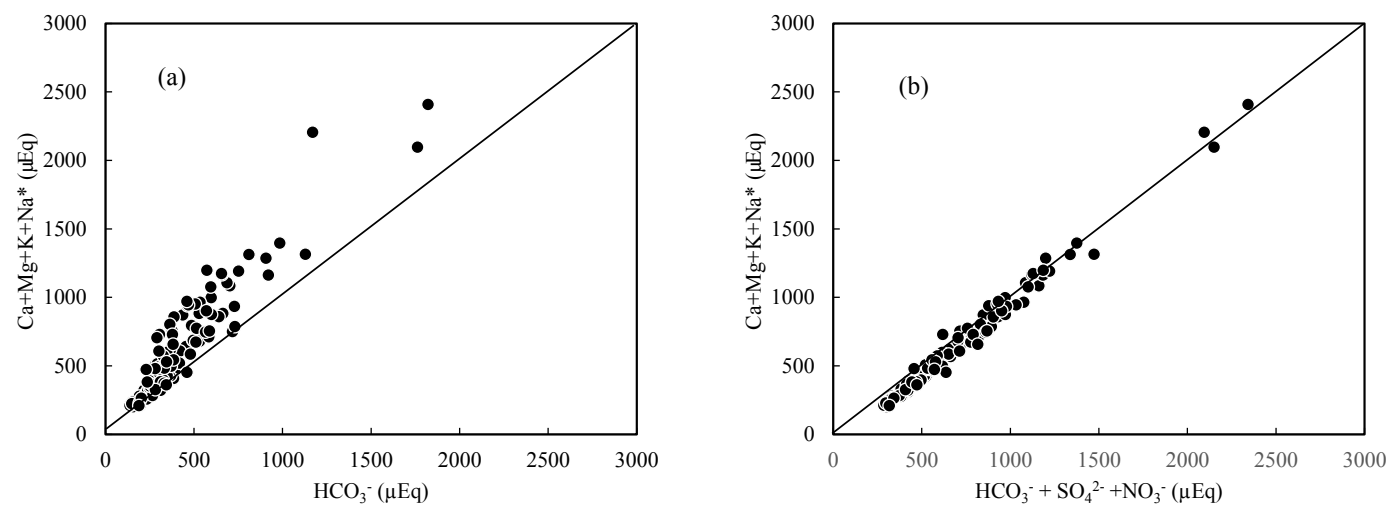

Figure 6. Plots of total cations derived from (a) carbonate and silicate weathering vs. $\mathrm{HCO}_{3}^{-}$and (b) $\mathrm{HCO}_{3}^{-}+\mathrm{SO}_{4}^{2-}+\mathrm{NO}_{3}^{-}$for river water in the SECRB.

$\left(66.6 \times 10^{3} \mathrm{~mol} \mathrm{~km}^{-2} \mathrm{a}^{-1}\right.$, Liu et al., 2013). The high $\mathrm{CO}_{2}$ consumption rates by silicate weathering in the SECRB could be attributed to extensive distribution of silicate rocks, high runoff and favorable climatic conditions. The regional fluxes of $\mathrm{CO}_{2}$ consumption by silicate and carbonate weathering are about $47.9 \times 10^{9} \mathrm{~mol} \mathrm{a}^{-1}\left(0.57 \times 10^{12} \mathrm{~g} \mathrm{C} \mathrm{a}^{-1}\right)$ and $41.9 \times 10^{9} \mathrm{~mol} \mathrm{a}^{-1}\left(0.50 \times 10^{12} \mathrm{~g} \mathrm{Ca}^{-1}\right)$ in the whole SECRB.

However, in addition to $\mathrm{CO}_{2}$, the anthropogenic-sourced proton (e.g., $\mathrm{H}_{2} \mathrm{SO}_{4}$ and $\mathrm{HNO}_{3}$ ) is well documented as significant proton providers in rock weathering processes (Galy and France-Lanord, 1999; Karim and Veizer, 2000; Yoshimura et al., 2001; Han and Liu, 2004; Spence and Telmer, 2005; Lerman and $\mathrm{Wu}, 2006$; $\mathrm{Xu}$ and Liu, 2007, 2010; Perrin et al., 2008; Gandois et al., 2011). Sulfuric acid can be generated by natural oxidation of pyrite and anthropogenic emissions of $\mathrm{SO}_{2}$ from coal combustion and subsequently dissolve carbonate and silicate minerals. The riverine nitrate in a watershed can be derived from atmospheric deposition, synthetic fertilizers, microbial nitrification, sewage and manure, etc. (e.g., Kendall, 1998). Although it is difficult to determine the sources of nitrate in river water, we can at least simply assume that nitrate from acid deposition is one of the proton providers. The consumption of $\mathrm{CO}_{2}$ by rock weathering would be overestimated if $\mathrm{H}_{2} \mathrm{SO}_{4}$ - and $\mathrm{HNO}_{3}$-induced rock weathering was ignored (Spence and Telmer, 2005; Xu and Liu, 2010; Shin et al., 2011; Gandois et al., 2011). Thus, the role that anthropogenic-sourced protons play on chemical weathering is crucial for an accurate estimation of $\mathrm{CO}_{2}$ consumption by rock weathering.

Rapid economic growth and increased energy needs have resulted in severe air pollution problems in many areas of China, indicated by high levels of mineral acids (predominately sulfuric) observed in precipitation (Larssen and Carmichael, 2000; Pan et al., 2013; L. Liu et al., 2016). The national $\mathrm{SO}_{2}$ emissions in 2010 reached $30.8 \mathrm{Tg} \mathrm{a}^{-1}$ (Lu et al., 2011). Previous studies documented that fossil fuel combustion accounts for the most sulfur deposition $(\sim 77 \%)$ in
China (L. Liu et al., 2016). The wet deposition rate of nitrogen is the highest over central and southern China, with mean values of 20.2, 18.2 and $25.8 \mathrm{~kg} \mathrm{~N} \mathrm{ha}^{-1} \mathrm{a}^{-1}$ in Zhejiang, Fujian and Guangdong provinces, respectively ( $\mathrm{Lu}$ and Tian, 2007). Current sulfur and nitrogen depositions in the southeastern coastal region are still among the highest in China (Fang et al., 2013; Cui et al., 2014; L. Liu et al., 2016).

The involvement of protons originating from $\mathrm{H}_{2} \mathrm{SO}_{4}$ and $\mathrm{HNO}_{3}$ in the river water can be illustrated by the stoichiometry between cations and anions, shown in Fig. 6 . In the rivers of the SECRB, the sum of the cations released by silicate and carbonate weathering could not be balanced by $\mathrm{HCO}_{3}^{-}$ only (Fig. 6a), but were almost balanced by the sum of $\mathrm{HCO}_{3}^{-}, \mathrm{SO}_{4}^{2-}$ and $\mathrm{NO}_{3}^{-}$(Fig. 6b). This implies that $\mathrm{H}_{2} \mathrm{CO}_{3}$, $\mathrm{H}_{2} \mathrm{SO}_{4}$ and $\mathrm{HNO}_{3}$ are the potential erosion agents in chemical weathering in the SECRB. The $\delta^{13} \mathrm{C}$ values of the water samples showed a wide range, from $-11.0 \%$ to $-24.3 \%$, with an average of $-19.4 \%$. The $\delta^{13} \mathrm{C}$ from soil is dominated by the relative contribution from $\mathrm{C}_{3}$ and $\mathrm{C}_{4}$ plants (Das et al., 2005). The studied areas have subtropical temperatures and humidity, and thus $\mathrm{C}_{3}$ processes are dominant. The $\delta^{13} \mathrm{C}$ of soil $\mathrm{CO}_{2}$ is derived primarily from $\delta^{13} \mathrm{C}$ of organic material, which typically has a value between $-24 \%$ and $-34 \%$, with an average of $-28 \%$ (Faure, 1986). According to previous studies, the average value for $\mathrm{C}_{3}$ trees and shrubs are from $-24.4 \%$ o to $-30.5 \%$, and most of them are lower than $-28 \%$ in southern China (Chen et al., 2005; Xiang, 2006; Dou et al., 2013). After accounting for the isotopic effect from the diffusion of $\mathrm{CO}_{2}$ from soil, the resulted $\delta^{13} \mathrm{C}$ (from the terrestrial $\mathrm{C}_{3}$ plant process) should be $\sim-25 \%$ (Cerling et al., 1991). This mean DIC derived from silicate weathering by carbonic acid $\left(100 \%\right.$ from soil $\left.\mathrm{CO}_{2}\right)$ would yield a $\delta^{13} \mathrm{C}$ value of $-25 \%$. Carbonate rocks are generally derived from marine systems and typically have a $\delta^{13} \mathrm{C}$ value close to zero (Das et al., 2005). Thus, the theoretical $\delta^{13} \mathrm{C}$ value of DIC derived from carbonate weathering by carbonic acid $(50 \%$ from soil $\mathrm{CO}_{2}$ and $50 \%$ from carbonate rocks) is $-12.5 \%$. DIC derived from carbonate weathering by sulfuric acid are 

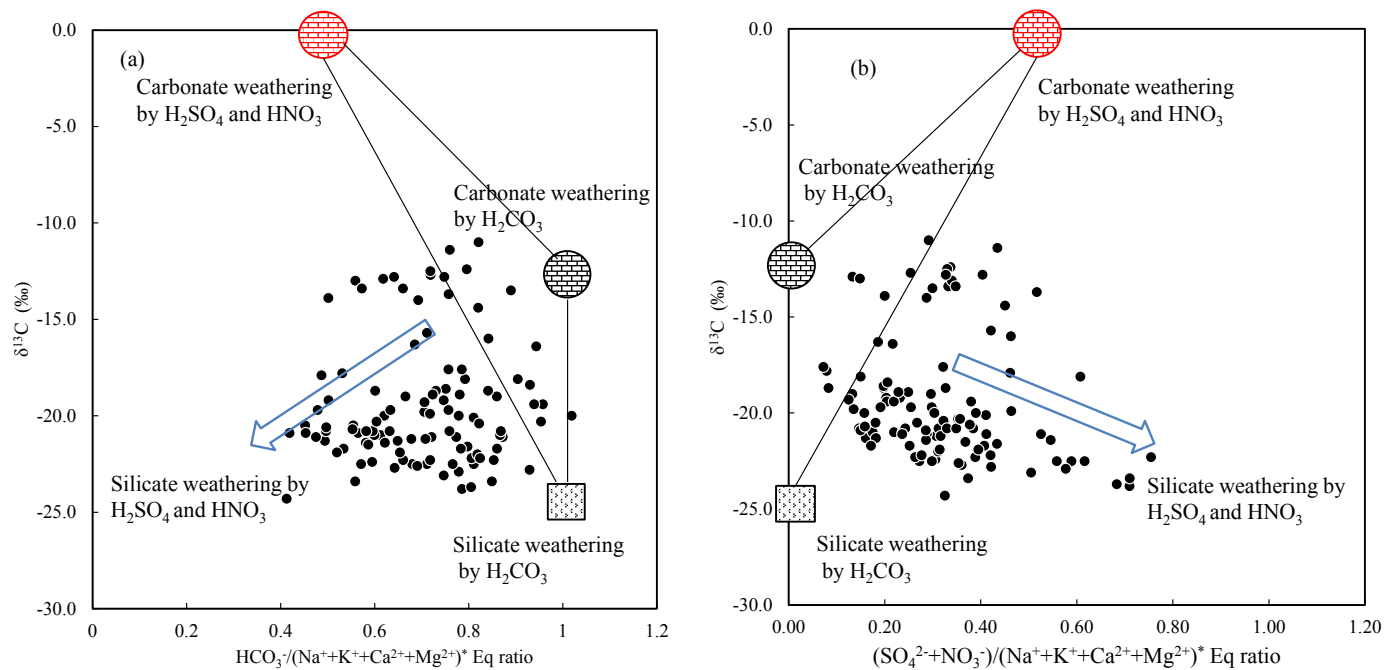

Figure 7. (a) $\delta^{13} \mathrm{C}_{\mathrm{DIC}}$ vs. $\mathrm{HCO}_{3}^{-} /\left(\mathrm{Na}^{+}+\mathrm{K}^{+}+\mathrm{Ca}^{2+}+\mathrm{Mg}^{2+}\right)^{*}$ and (b) $\left(\mathrm{SO}_{4}^{2-}+\mathrm{NO}_{3}^{-}\right) /\left(\mathrm{Na}^{+}+\mathrm{K}^{+}+\mathrm{Ca}^{2+}+\mathrm{Mg}^{2+}\right)^{*}$ equivalent ratio in river water draining the SECRB $(*$ noted concentrations corrected for atmospheric and anthropogenic inputs). The plots show that most water deviates from the three end-member mixing areas (carbonate weathering by carbonic acid and sulfuric/nitric acid and silicate weathering by carbonic acid).

all from carbonate rocks; thus the $\delta^{13} \mathrm{C}$ of the DIC would be $0 \%$. Based on the above discussion, sources of riverine DIC from different end-members in the SECRB were plotted in Fig. 7. Most water samples drift away from the three end-member mixing areas (carbonate and silicate weathering by carbonic acid and carbonate weathering by sulfuric acid) and towards silicate weathering by sulfuric and nitric acid area, clearly illustrating the effect of the anthropogenicsourced protons (sulfuric and nitric acid) on silicate weathering in the SECRB.

Considering the $\mathrm{H}_{2} \mathrm{SO}_{4}$ and $\mathrm{HNO}_{3}$ effects on chemical weathering, $\mathrm{CO}_{2}$ consumption by silicate weathering can be determined from the equation below (Moon et al., 2007; Ryu et al., 2008; Shin et al., 2011):

$$
\begin{aligned}
{\left[\mathrm{CO}_{2}\right]_{\text {SNSW }}=} & {[\mathrm{Na}]_{\text {sil }}+[\mathrm{K}]_{\text {sil }}+2[\mathrm{Ca}]_{\text {sil }}+2[\mathrm{Mg}]_{\text {sil }} } \\
& -\gamma \times\left[2 \mathrm{SO}_{4}+\mathrm{NO}_{3}\right]_{\text {atmos }},
\end{aligned}
$$

where $\gamma$ is calculated by cation ${ }_{\text {sil }} /$ (cation $_{\text {sil }}+$ cation $\left._{\text {carb }}\right)$.

Based on the calculation in Sect. 5.1, $\mathrm{SO}_{4}^{2-}$ and $\mathrm{NO}_{3}^{-}$ in river water were mainly derived from atmospheric inputs. Assuming that $\mathrm{SO}_{4}^{2-}$ and $\mathrm{NO}_{3}^{-}$in river water derived from atmospheric input (after correction for sea-salt contribution) are all from acid precipitation and considering $\mathrm{H}_{2} \mathrm{SO}_{4}$ and $\mathrm{HNO}_{3}$ effects, $\mathrm{CO}_{2}$ consumption rates by silicate weathering (SNSW) are estimated between $55 \times 10^{3}$ and $286 \times 10^{3} \mathrm{~mol} \mathrm{~km}^{-2} \mathrm{a}^{-1}$ for major river watersheds in the SECRB. For the whole SECRB, the actual $\mathrm{CO}_{2}$ consumption rate by silicate is $191 \times 10^{3} \mathrm{~mol} \mathrm{~km}^{-2} \mathrm{a}^{-1}$ when the effect of $\mathrm{H}_{2} \mathrm{SO}_{4}$ and $\mathrm{HNO}_{3}$ is considered. The flux of $\mathrm{CO}_{2}$ consumption is overestimated by $16.1 \times 10^{9} \mathrm{~mol} \mathrm{a}^{-1}$ $\left(0.19 \times 10^{12} \mathrm{~g} \mathrm{Ca}^{-1}\right)$ due to the involvement of $\mathrm{H}_{2} \mathrm{SO}_{4}$ and $\mathrm{HNO}_{3}$ from acid precipitation, accounting for approximately
$33.6 \%$ of the total $\mathrm{CO}_{2}$ consumption flux by silicate weathering in the SECRB. It highlights the fact that the drawdown of atmospheric $\mathrm{CO}_{2}$ by silicate weathering can be significantly overestimated if acid deposition is ignored in long-term perspectives. The result quantitatively shows that anthropogenic activities can significantly affect rock weathering and associated atmospheric $\mathrm{CO}_{2}$ consumption. The quantification of this effect needs to be well evaluated in Asia and globally, taking into account current and future human activity.

It is noticeable that the chemical weathering and associated $\mathrm{CO}_{2}$ consumption rates for the study area were calculated by the river water geochemistry of high-flow season. As a subtropical monsoon climate area, the river water of the southeastern coastal rivers is mainly recharged by rain, and the amount of precipitation in the high-flow season accounts for more than $70 \%$ of annual precipitation in the area. The processes in the low-flow season might be different to some extent. It is worth making further efforts to investigate the hydrological and temperature effects on weathering rate and flux, as well as evaluate the anthropogenic impact in different climate regimes and hydrology seasons.

\section{Conclusions}

River water in the southeastern coastal region of China is characterized by high proportions of $\mathrm{Na}^{+}, \mathrm{K}^{+}$and dissolved $\mathrm{SiO}_{2}$, indicating that the water chemistry of the rivers in the SECRB is mainly controlled by silicate weathering. The dissolved cationic loads of the rivers in the study area originate dominantly from silicate weathering, which accounts for $39.5 \%(17.8 \%-74.0 \%)$ of the total cationic loads. Carbon- 
ate weathering, atmospheric and anthropogenic inputs account for $30.6 \%, 14.3 \%$ and $15.7 \%$, respectively. Meanwhile, more than $70 \%$ of $\mathrm{SO}_{4}^{2-}$ and $\mathrm{NO}_{3}^{-}$in the river water is derived from atmospheric input. The chemical weathering rate of silicates and carbonates for the whole SECRB are estimated to be approximately 23.7 and $24.5 \mathrm{t} \mathrm{km}^{-2} \mathrm{a}^{-1}$. About $8.04 \times 10^{6} \mathrm{ta}^{-1}$ of dissolved solids originating from rock weathering are transported into the East and South China seas by these rivers in the SECRB. With the assumption that all the protons involved in the weathering reaction are provided by carbonic acid, the $\mathrm{CO}_{2}$ consumption rates by silicate and carbonate weathering are 287 and $251 \times 10^{3} \mathrm{~mol} \mathrm{~km}^{-2} \mathrm{a}^{-1}$, respectively. However, both water chemistry and carbon isotope data provide solid evidence that sulfuric and nitric acid from acid precipitation serve as significant agents during chemical weathering. Considering the effect of sulfuric and nitric acid, the $\mathrm{CO}_{2}$ consumption rate by silicate weathering for the SECRB is $191 \times 10^{3} \mathrm{~mol} \mathrm{~km}^{-2} \mathrm{a}^{-1}$. Therefore, the $\mathrm{CO}_{2}$ consumption flux would be overestimated by $16.1 \times 10^{9} \mathrm{~mol} \mathrm{a}^{-1}(0.19 \times$ $10^{12} \mathrm{~g} \mathrm{Ca}^{-1}$ ) in the SECRB if the effect of sulfuric and nitric acid is ignored. This work quantitatively illustrates that anthropogenic disturbance by acid precipitation has a profound impact on $\mathrm{CO}_{2}$ sequestration by rock weathering.

Data availability. The data in this study have been presented in the tables of the article and can also be requested from the corresponding author.

Author contributions. WL and ZX designed the study. WL, HS, TZ and CS carried out the study. WL, ZX, HS and TL contributed to data treatment and calculations. WL and ZX prepared the manuscript with contributions from all co-authors.

Competing interests. The authors declare that they have no conflict of interest.

Special issue statement. This article is part of the special issue "Human impacts on carbon fluxes in Asian river systems". It is not associated with a conference.

Acknowledgements. This work was financially supported by the Strategic Priority Research Program of Chinese Academy of Sciences (grant nos. XDB26000000 and XDB15010405), and Natural Science Foundation of China (grant no. 41673020, 91747202, 41772380 and 41730857).

Edited by: V. V. S. S. Sarma

Reviewed by: Shouye Yang and two anonymous referees

\section{References}

Amiotte-Suchet, P., Probst, A., and Probst, J.-L.: Influence of acid rain on $\mathrm{CO}_{2}$ consumption by rock weathering: local and global scales, Water Air Soil Pollut., 85, 1563-1568, 1995.

Amiotte-Suchet, P., Probst, J.-L., and Ludwig, W.: Worldwide distribution of continental rock lithology: implications for the atmospheric/soil $\mathrm{CO}_{2}$ uptake by continental weathering and alkalinity river transport to the oceans, Global Biogeochem. Cy., 17, 10381052, 2003.

Bai, H., Song, L. S., and Xia, W. P.: Prospect analysis of hot dry rock (HDR) in Eastern part of Jiangxi province, Coal Geol. China, 26, 41-44, 2014 (in Chinese).

Berner, R. A. and Caldeira, K.: The need for mass balance and feedback in the geochemical carbon cycle, Geology, 25, 955-956, 1997.

Bluth, G. J. S. and Kump, L. R.: Lithological and climatological controls of river chemistry, Geochim. Cosmochim. Acta, 58, 2341-2359, 1994.

Brantley, S. L. and Lebedeva, M.: Learning to read the chemistry of regolith to understand the Critical Zone, Annu. Rev. Earth Pl. Sc., 39, 387-416, 2011.

Calmels, D., Gaillardet, J., Brenot, A., and France-Lanord, C. Sustained sulfide oxidation by physical erosion processes in the Mackenzie River basin: climatic perspectives, Geology, 35, 1003-1006, 2007.

Calmels, D., Galy, A., Hovius, N., Bickle, M., West, A. J., Chen, M.-C., and Chapman, H.: Contribution of deep groundwater to the weathering budget in a rapidly eroding mountain belt, Taiwan, Earth Planet. Sc. Lett., 303, 48-58, 2011.

Cerling, T. E., Solomon, D. K., Quade, J., and Bownman, J. R.: On the isotopic composition of soil $\mathrm{CO}_{2}$, Geochim. Cosmochim. Acta, 55, 3403-3405, 1991.

Chen, Q., Shen, C., Sun, Y., Peng, S., Yi, W., Li Z., and Jiang, M.: Spatial and temporal distribution of carbon isotopes in soil organic matter at the Dinghushan Biosphere Reserve, South China, Plant Soil, 273, 115-128, 2005.

Cheng, Y., Liu, Y., Huo, M., Sun, Q., Wang, H., Chen, Z., and Bai, Y.: Chemical characteristics of precipitation at Nanping Mangdang Mountain in eastern China during spring, J. Environ. Sci., 23, 1350-1358, 2011.

Chetelat, B., Liu, C., Zhao, Z., Wang, Q., Li, S., Li, J., and Wang, B.: Geochemistry of the dissolved load of the Changjiang Basin rivers: anthropogenic impacts and chemical weathering, Geochim. Cosmochim. Acta, 72, 4254-4277, 2008.

Cui, J., Zhou, J., Peng, Y., He, Y., Yang, H., and Mao, J.: Atmospheric wet deposition of nitrogen and sulfur to a typical red soil agroecosystem in Southeast China during the ten-year monsoon seasons (2003-2012). Atmos. Environ. 82, 121-129, 2014.

Das, A., Krishnaswami, S., Sarin, M. M., and Pande, K.: Chemical weathering in the Krishna Basin and Western Ghats of the Deccan Traps, India: Rates of basalt weathering and their controls, Geochim. Cosmochim. Acta, 69, 2067-2084, 2005.

Dou, X., Deng, Q., Li, M., Wang, W., Zhang, Q., and Cheng, X.: Reforestation of Pinus massoniana alters soil organic carbon and nitrogen dynamics in eroded soil in south China, Ecol. Eng., 52, 154-160, 2013.

Edmond, J. M., Palmer, M. R., Measures, C. I., Grant, B., and Stallard, R. F.: The fluvial geochemistry and denudation rate of the 
Guayana Shield in Venezuela, Colombia, and Brazil, Geochim. Cosmochim. Acta, 59, 3301-3325, 1995.

Fan, B. L, Zhao, Z. Q., Tao, F. X., Liu, B. J., Tao, Z. H., Gao, S., and He, M. Y.: Characteristics of carbonate, evaporite and silicate weathering in Huanghe River basin: A comparison among the upstream, midstream and downstream, J. Asian Earth Sci., 96, 17-26, 2014.

Fang, Y., Wang, X., Zhu, F., Wu, Z., Li, J., Zhong, L., Chen, D., and Yoh, M.: Three-decade changes in chemical composition of precipitation in Guangzhou city, southern China: has precipitation recovered from acidification following sulphur dioxide emission control?, Tellus B, 65, 1-15, 2013.

Faure, G.: Principles of Isotope Geology, Wiley, Toronto, 492-493, 1986.

Fujian Bureau of Geology and Mineral Resources (FJBGMR): Regional Geology of Fujian Province, Geol. Publ. House, Beijing, p. 671, 1985 (in Chinese with English abstract).

Gaillardet, J., Dupré, B., Allègre, C. J., and Négrel, P.: Chemical and physical denudation in the Amazon River basin, Chem. Geol., 142, 141-173, 1997.

Gaillardet, J., Dupré, B., Louvat, P., and Allegre, C. J.: Global silicate weathering and $\mathrm{CO}_{2}$ consumption rates deduced from the chemistry of large rivers, Chem. Geol., 159, 3-30, 1999.

Galy, A. and France-Lanord, C.: Weathering processes in the Ganges-Brahmaputra basin and the riverine alkalinity budget, Chem. Geol., 159, 31-60, 1999.

Gandois, L., Perrin, A.-S., and Probst, A.: Impact of nitrogenous fertilizer-induced proton release on cultivated soils with contrasting carbonate contents: A column experiment, Geochim. Cosmochim. Acta, 75, 1185-1198, 2011.

Gislason, S. R., Arnorsson, S., and Armannsson, H.: Chemical weathering of basalt in southwest Iceland: effects of runoff, age of rocks and vegetative/glacial cover, Am. J. Sci., 296, 837-907, 1996.

Han, G. and Liu, C. Q.: Water geochemistry controlled by carbonate dissolution: a study of the river waters draining karst-dominated terrain, Guizhou Province, China, Chem. Geol., 204, 1-21, 2004.

Han, Z. W., Ueda, H., and Sakurai, T.: Model study on acidifying wet deposition in East Asia during wintertime, Atmos. Environ., 40, 2360-2373, 2006.

Hartmann, J., Jansen, N., Dürr, H. H., Kempe, S., and Köhler, P.: Global $\mathrm{CO}_{2}$ consumption by chemical weathering: what is the contribution of highly active weathering regions?, Global Planet. Change, 69, 185-194, 2009.

Huang, K., Zhuang, G., Xu, C., Wang Y., and Tang A.: The chemistry of the severe acidic precipitation in Shanghai, China, Atmos. Res., 89, 149-160, 2008.

Huh, Y. S.: Chemical weathering and climate - a global experiment: a review, Geosci. J., 7, 277-288, 2003.

Hydrology Bureau of the Ministry of Water Resources of People's Republic of China: Hydrological data of river basins in Zhejiang, Fujian province and Taiwan region, Annual Hydrological Report of 2010, P. R. China, vol. 7, 2011 (in Chinese).

Ji, H. and Jiang, Y.: Carbon flux and C, S isotopic characteristics of river waters from a karstic and a granitic terrain in the Yangtze River system, J. Asian Earth Sci., 57, 38-53, 2012.

Karim, A. and Veizer, H. E.: Weathering processes in the Indus River Basin: implication from riverine carbon, sulphur, oxygen and strontium isotopes, Chem. Geol., 170, 153-177, 2000.
Kendall, C.: Tracing nitrogen sources and cycling in catchments, in: Isotope Tracers in Catchment Hydrology, edited by: Kendall, $\mathrm{C}$. and McDonnell, J. J., Elsevier, Amsterdam, 1998.

Kump, L. R., Brantley, S. L., and Arthur, M. A.: Chemical weathering, atmospheric $\mathrm{CO}_{2}$ and climate, Ann. Rev. Earth Planet. Sci., 28, 611-667, 2000.

Larssen, T. and Carmichael, G. R.: Acid rain and acidification in China: the importance of base cation deposition, Environ. Poll., 110, 89-102, 2000.

Larssen, T., Lydersen, E., Tang, D., He, Y., Gao, J., Liu, H., Duan, L., and Seip, H. M.: Acid rain in China, Environ. Sci. Technol., 40, 418-425, 2006.

Lerman, A. and $\mathrm{Wu}, \mathrm{L} .: \mathrm{CO}_{2}$ and sulfuric acid controls of weathering and river water composition, J. Geochem. Explor., 88, 427430, 2006.

Lerman, A., Wu, L., and Mackenzie, F. T.: $\mathrm{CO}_{2}$ and $\mathrm{H}_{2} \mathrm{SO}_{4}$ consumption in weathering and material transport to the ocean, and their role in the global carbon balance, Mar. Chem., 106, 326$350,2007$.

Li, S. and Bush, R. T.: Changing fluxes of carbon and other solutes from the Mekong River, Sci. Rep., 5, 16005, https://doi.org/10.1038/srep16005, 2015.

Li, S. Y., Xu, Z. F., Wang, H., Wang, J. H., and Zhang, Q. F.: Geochemistry of the upper Han River basin, China. 3: anthropogenic inputs and chemical weathering to the dissolved load, Chem. Geol., 264, 89-95, 2009.

Li, S. Y., Lu, X. X., He, M., Zhou, Y., Bei, R., Li, L., and Zieglera, A. D.: Major element chemistry in the Upper Yangtze River: a case study of the Longchuanjiang River, Geomorphology, 129, 29-42, 2011.

Li, S. Y., Lu, X. X., and Bush, R. T.: Chemical weathering and $\mathrm{CO}_{2}$ consumption in the Lower Mekong River, Sci. Total Environ., 472, 162-177, 2014.

Liu, B., Liu, C.-Q., Zhang, G., Zhao, Z.-Q., Li, S.-L., Hu, J., Ding, H., Lang, Y.-C., and Li, X.-D.: Chemical weathering under midto cool temperate and monsoon-controlled climate: A study on water geochemistry of the Songhuajiang River system, northeast China, Appl. Geochem., 31, 265-278, 2013.

Liu, L., Zhang, X., Wang, S., Zhang, W., and Lu, X.: Bulk sulfur (S) deposition in China, Atmos. Environ., 135, 41-49, 2016.

Liu, W., Shi, C., Xu, Z., Zhao, T., Jiang, H., Liang, C., Zhang, X., Zhou, Li, and Yu, C.: Water geochemistry of the Qiantangjiang River, East China: Chemical weathering and $\mathrm{CO}_{2}$ consumption in a basin affected by severe acid deposition, J. Asian Earth Sci., 127, 246-256, 2016.

Lu, C. and Tian, H.: Spatial and temporal patterns of nitrogen deposition in China: Synthesis of observational data, J. Geophys. Res., 112, D22S05, https://doi.org/10.1029/2006JD007990, 2007.

Lu, Z., Zhang, Q., and Streets, D. G.: Sulfur dioxide and primary carbonaceous aerosol emissions in China and India, 1996-2010, Atmos. Chem. Phys., 11, 9839-9864, https://doi.org/10.5194/acp-11-9839-2011, 2011.

Millot, R., Gaillardet, J., Dupré, B., and Allègre, C. J.: The global control of silicate weathering rates and the coupling with physical erosion: new insights from rivers of the Canadian Shield, Earth Planet. Sc. Lett., 196, 83-98, 2002.

Millot, R., Gaillardet, J., Dupré, B., and Allègre, C. J.: Northern latitude chemical weathering rates: clues from the Mackenzie 
River Basin, Canada, Geochim. Cosmochim. Acta, 67, 13051329, 2003.

Ministry of Environmental Protection of China: China Environmental Quality Report 2008, China Environmental Sciences Press, Beijing, 2009 (in Chinese).

Moon, S., Huh, Y., Qin, J., and van Pho, N.: Chemical weathering in the Hong (Red) River basin: Rates of silicate weathering and their controlling factors, Geochim. Cosmochim. Acta, 71, 14111430, 2007.

Moon, S., Huh, Y., and Zaitsev, A.: Hydrochemistry of the Amur River: Weathering in a Northern Temperate Basin, Aquat. Geochem., 15, 497-527, 2009.

Moon, S., Chamberlain, C. P., and Hilley, G. E.: New estimates of silicate weathering rates and their uncertainties in global rivers, Geochim. Cosmochim. Acta, 134, 257-274, 2014.

Mortatti, J. and Probst, J. L.: Silicate rock weathering and atmospheric/soil $\mathrm{CO}_{2}$ uptake in the Amazon basin estimated from river water geochemistry: seasonal and spatial variations, Chem. Geol., 197, 177-196, 2003.

Négrel, P., Allègre, C. J., Dupré, B., and Lewin, E.: Erosion sources determined by inversion of major and trace element ratios and strontium isotopic ratios in river water: the Congo Basin Case, Earth Planet. Sc. Lett., 120, 59-76, 1993.

Noh, H., Huh, Y., Qin, J., and Ellis, A.: Chemical weathering in the Three Rivers region of Eastern Tibet, Geochim. Cosmochim. Acta, 73, 1857-1877, 2009.

Oliva, P., Viers, J., and Dupré B.: Chemical weathering in granitic environments, Chem. Geol., 202, 225-256, 2003.

Pan, Y. P., Wang, Y. S., Tang, G. Q., and Wu, D.: Spatial distribution and temporal variations of atmospheric sulfur deposition in Northern China: insights into the potential acidification risks, Atmos. Chem. Phys., 13, 1675-1688, https://doi.org/10.5194/acp13-1675-2013, 2013.

Pepin, E., Guyot, J. L., Armijos, E., Bazan, H., Fraizy, P., Moquet, J. S., Noriega, L., Lavado, W., Pombosa, R., and Vauchel, P.: Climatic control on eastern Andean denudation rates (Central Cordillera from Ecuador to Bolivia), J. S. Am. Earth Sci., 44, 85-93, 2013.

Perrin, A.-S., Probst, A., and Probst, J.-L.: Impact of nitrogenous fertilizers on carbonate dissolution in small agricultural catchments: Implications for weathering $\mathrm{CO}_{2}$ uptake at regional and global scales, Geochim. Cosmochim. Acta, 72, 3105-3123, 2008.

Probst, A., Gh'mari, A. El., Aubert, D., Fritz, B., and McNutt, R.: Strontium as a tracer of weathering processes in a silicate catchment polluted by acid atmospheric inputs, Strengbach, France, Chem. Geol., 170, 203-219, 2000.

Raymo, M. E. and Ruddiman, W. F.: Tectonic forcing of late Cenozoic climate, Nature, 359, 117-122, 1992.

Raymond, P. A., Oh, N. H., Turner, R. E., and Broussard, W.: Anthropogenically enhanced fluxes of water and carbon from the Mississippi River, Nature, 451, 449-452, 2008.

Regnier, P., Friedlingstein, P., Ciais, P., Mackenzie, F. T., Gruber, N., Janssens, I. A., Laruelle, G. G., Lauerwald, R., Luyssaert, S., Andersson, A. J., Arndt, S., Arnosti, C., Borges, A. V., Dale, A. W., Gallego-Sala, A., Goddéris, Y., Goossens, N., Hartmann, J., Heinze, C., Ilyina, T., Joos, F., LaRowe, D. E., Leifeld, J., Meysman, F. J. R., Munhoven, G., Raymond, P. A., Spahni, R., Suntharalingam, P., and Thullner, M.: Anthropogenic perturba- tion of the carbon fluxes from land to ocean, Nat. Geosci., 6 , 597-607, 2013.

Reuss, J. O., Cosby, B. J., and Wright, R. F.: Chemical processes governing soil and water acidification, Nature, 329, 27-32, 1987.

Roy, S., Gaillardet, J., and Allègre, C. J.: Geochemistry of dissolved and suspended loads of the Seine river, France: anthropogenic impact, carbonate and silicate weathering, Geochim. Cosmochim. Acta, 63, 1277-1292, 1999.

Ryu, J. S., Lee, K. S., Chang, H. W., and Shin, H. S.: Chemical weathering of carbonates and silicates in the Han River basin, South Korea, Chem. Geol. 247, 66-80, 2008.

Semhi, K., Amiotte Suchet, P., Clauer, N., and Probst, J.-L.: Impact of nitrogen fertilizers on the natural weathering-erosion processes and fluvial transport in the Garonne basin, Appl. Geochem., 15, 865-874, 2000.

Shi, C.: Chemical characteristics and weathering of rivers in the Coast of Southeast China, PhD Thesis, Institute of Geology and Geophysics, Chinese academy of sciences, Beijing, China, 1100, 2014 (in Chinese with English abstract).

Shin, W.-J., Ryu, J.-S., Park, Y., and Lee, K.-S.: Chemical weathering and associated $\mathrm{CO}_{2}$ consumption in six major river basins, South Korea, Geomorphology, 129, 334-341, 2011.

Shu, L. S., Zhou, X. M., Deng, P., Wang, B., Jiang, S. Y., Yu, J. H., and Zhao, X. X.: Mesozoic tectonic evolution of the Southeast China Block: New insights from basin analysis, J. Asian Earth Sci., 34, 376-391, 2009.

Spence, J. and Telmer, K.: The role of sulfur in chemical weathering and atmospheric $\mathrm{CO}_{2}$ fluxes: Evidence from major ions, $\delta^{13} \mathrm{C}_{\text {DIC }}$, and ${ }^{34} \mathrm{~S}_{\mathrm{SO}_{4}}$ in rivers of the Canadian Cordillera, Geochim. Cosmochim. Acta, 69, 5441-5458, 2005.

Stallard, R. F. and Edmond, J. M.: Geochemistry of the Amazon, Precipitation chemistry and the marine contribution to the dissolved load at the time of peak discharge, J. Geophys. Res., 86, 9844-9858, 1981.

U.S. National Research Council Committee: Basic Research Opportunities in Earth Science, Washington, DC, Nat. Acad., 154 pp., 2001.

Viers, J., Dupré, B., Braun, J. J., Freydier, R., Greenberg, S., Ngoupayou, J. N., and Nkamdjou, L. S.: Evidence for nonconservative behavior of chlorine in humid tropical environments, Aquat. Geochem., 7, 127-154, 2001.

Vries, W. D., Reinds, G. J., and Vel, E.: Intensive monitoring of forest ecosystems in Europe. 2-Atmospheric deposition and its impacts on soil solution chemistry, For. Ecol. Manage., 174, 97115, 2003.

Wang, T. J., Jin, L. S., Li, Z. K., and Lam, K. S.: A modeling study on acid rain and recommended emission control strategies in China, Atmos. Environ., 34, 4467-4477, 2000.

West, A. J., Galy, A., and Bickle, M.: Tectonic and climatic controls on silicate weathering, Earth Planet. Sc. Lett., 235, 211228, 2005.

Wu, W., Zheng, H., Yang, J., Luo, C., and Zhou, B.: Chemical weathering, atmospheric $\mathrm{CO}_{2}$ consumption, and the controlling factors in a subtropical metamorphic-hosted watershed, Chem. Geol., 356, 141-150, 2013.

Xiang, L.: Study on Coupling between Water and Carbon of Artificial Forests Communities in Subtropical Southern China, Master Dissertation, Institute of Geographical Sciences and Natural 
Sources, Chinese Academy of Sciences, China, 2006 (in Chinese).

Xu, H., Bi, X.-H., Feng, Y.-C., Lin, F.-M., Jiao, L., Hong, S.-M., Liu, W.-G., and Zhang, X.-Y.: Chemical composition of precipitation and its sources in Hangzhou, China, Environ. Monit. Assess., 183, 581-592, 2011.

$\mathrm{Xu}$, Y., Wang, C. Y., and Zhao, T.: Using detrital zircons from river sands to constrain major tectono-thermal events of the Cathaysia Block, SE China, J. Asian Earth Sci., 124, 1-13, 2016.

$\mathrm{Xu}, \mathrm{Z}$. and Liu, C.-Q.: Chemical weathering in the upper reaches of Xijiang River draining the Yunnan-Guizhou Plateau, Southwest China, Chem. Geol., 239, 83-95, 2007.

$\mathrm{Xu}, \mathrm{Z}$. and Liu, C.-Q.: Water geochemistry of the Xijiang basin rivers, South China: Chemical weathering and $\mathrm{CO}_{2}$ consumption, Appl. Geochem., 25, 1603-1614, 2010

Yoshimura, K., Nakao, S., Noto, M., Inokura, Y., Urata, K., Chen, M., and Lin, P. W.: Geochemical and stable isotope studies on natural water in the Taroko Gorge karst area, Taiwan - chemical weathering of carbonate rocks by deep source $\mathrm{CO}_{2}$ and sulfuric acid, Chem. Geol., 177, 415-430, 2001.

Zhang, M., Wang, S., Wu, F., Yuan, X., and Zhang, Y.: Chemical compositions of wet precipitation and anthropogenic influences at a developing urban site in southeastern China, Atmos. Res., 84, 311-322, 2007.
Zhang, S. R., Lu, X. X., Higgitt, D. L., Chen, C. T. A., Sun, H.-G., and Han, J. T.: Water chemistry of the Zhujiang (Pearl River): Natural processes and anthropogenic influences, J. Geophys. Res., 112, F01011, https://doi.org/10.1029/2006JF000493, 2007.

Zhao, W.: An analysis on the changing trend of acid rain and its causes in Fujian Province, Fujian Geogr., 19, 1-5, 2004 (in Chinese).

Zhou, X. M. and Li, W. X.: Origin of Late Mesozoic igneous rocks in Southeastern China: Implications for lithosphere subduction and underplating of mafic magmas, Tectonophysics, 326, 269$287,2000$.

Zhou, X. M., Sun, T., Shen, W. Z., Shu, L. S., and Niu, Y. L.: Petrogenesis of Mesozoic granitoids and volcanic rocks in South China: A response to tectonic evolution, Episodes, 29, 26-33, 2006.

Zhejiang Bureau of Geology and Mineral Resources (ZJBGMR): Regional Geology of Zhejiang Province, Geol. Publ. House, Beijing, p. 617 (in Chinese with English abstract), 1989. 